ARTICLE

DOI: $10.1038 / s 41467-018-06406-8$

\title{
Loss of pyruvate kinase M2 limits growth and triggers innate immune signaling in endothelial cells
}

Oliver A. Stone (10 1,9, Mohamed El-Brolosy (1) ${ }^{1}$, Kerstin Wilhelm², Xiaojing Liu ${ }^{3}$, Ana M. Romão ${ }^{4}$, Elisabetta Grillo ${ }^{5}$, Jason K.H. Lai ${ }^{1,10}{ }^{\text {, Stefan Günther }}{ }^{6}$, Sylvia Jeratsch7, Carsten Kuenne ${ }^{6}$, I-Ching Lee ${ }^{1}$, Thomas Braun ${ }^{4}$, Massimo M. Santoro ${ }^{8}$, Jason W. Locasale (i) ${ }^{3}$, Michael Potente ${ }^{2} \&$ Didier Y.R. Stainier ${ }^{1}$

Despite their inherent proximity to circulating oxygen and nutrients, endothelial cells (ECs) oxidize only a minor fraction of glucose in mitochondria, a metabolic specialization that is poorly understood. Here we show that the glycolytic enzyme pyruvate kinase M2 (PKM2) limits glucose oxidation, and maintains the growth and epigenetic state of ECs. We find that loss of PKM2 alters mitochondrial substrate utilization and impairs EC proliferation and migration in vivo. Mechanistically, we show that the NF-KB transcription factor RELB is responsive to PKM2 loss, limiting EC growth through the regulation of P53. Furthermore, Sadenosylmethionine synthesis is impaired in the absence of PKM2, resulting in DNA hypomethylation, de-repression of endogenous retroviral elements (ERVs) and activation of antiviral innate immune signalling. This work reveals the metabolic and functional consequences of glucose oxidation in the endothelium, highlights the importance of PKM2 for endothelial growth and links metabolic dysfunction with autoimmune activation in ECs.

\footnotetext{
${ }^{1}$ Department of Developmental Genetics, Max Planck Institute for Heart and Lung Research, Ludwigstrasse 43, 61231 Bad Nauheim, Germany.

${ }^{2}$ Angiogenesis \& Metabolism Laboratory, Max Planck Institute for Heart and Lung Research, Ludwigstrasse 43, 61231 Bad Nauheim, Germany. ${ }^{3}$ Department of Pharmacology and Cancer Biology, Duke University School of Medicine, Durham, NC 27710, USA. ${ }^{4}$ Department of Cardiac Development and Remodelling, Max Planck Institute for Heart and Lung Research, Ludwigstrasse 43, 61231 Bad Nauheim, Germany. ${ }^{5}$ Department of Oncology, KUL, Herestraat 49, 3000 Leuven, Belgium. ${ }^{6}$ ECCPS Bioinformatics and Deep Sequencing Platform, Max Planck Institute for Heart and Lung Research, Ludwigstrasse 43, 61231 Bad Nauheim, Germany. ${ }^{7}$ Biomolecular Mass Spectrometry, Max Planck Institute for Heart and Lung Research, Ludwigstrasse 43, 61231 Bad Nauheim, Germany. ${ }^{8}$ Department of Biology, University of Padua, Viale Giuseppe Colombo 3, 10141 Padua, Italy. ${ }^{9}$ Present address: Department of Physiology, Anatomy and Genetics, BHF Centre of Research Excellence, University of Oxford, Oxford OX1 3PT, UK. ${ }^{10}$ Present address: Mechanobiology Institute, National University of Singapore, Singapore 117411, Singapore. Correspondence and requests for materials should be addressed to O.A.S. (email: oliver.stone@dpag.ox.ac.uk)
} 
T he vertebrate vascular system comprises a vast and evolutionarily conserved network that supports tissue growth and homoeostasis ${ }^{1}$. Recent work has highlighted the high glycolytic rate of the endothelial cells (ECs) that line this network ${ }^{2}$, which under physiological conditions metabolize almost $90 \%$ of cellular glucose anaerobically to produce lactate ${ }^{3}$. The ability to generate ATP in this manner permits EC migration into non-perfused tissues and allows the expansion of vascular networks during organ growth ${ }^{2,4,5}$. Given these distinct metabolic traits, endothelial mitochondria have been considered to function largely as signalling organelles 6,7 , and the consequences of enhanced glucose oxidation in ECs are not well described. Recent work has shown that mitochondria play fundamental roles in EC growth and homoeostasis; $\beta$-oxidation of fatty acids is required for dNTP synthesis ${ }^{8}$, while glutamine metabolism is an essential source of TCA cycle intermediates that are necessary to support macromolecule biosynthesis in ECs ${ }^{3,9}$.

To gain insight into the functional and metabolic consequences of glucose oxidation in ECs, we analysed the function of the pyruvate kinase (PK) isozyme PKM2, which has been associated with aerobic glycolysis and growth in cancer cells ${ }^{10,11}$, and the maintenance of mitochondrial function in diabetic nephropathy ${ }^{12}$. Pyruvate kinase catalyses the final step in glycolysis, generating pyruvate and ATP from phosphoenolpyruvate and $\mathrm{ADP}^{13}$. In higher vertebrates, two genes (PKLR and $\left.P K M\right)$ encode four PK isozymes that vary in their expression patterns and activity ${ }^{14}$. Alternative splicing of the $P K M$ gene to include exon 9 or 10 generates the PKM1 and PKM2 isozymes, respectively ${ }^{15}$. While PKM1 exhibits constitutively high PK activity and its expression is associated with a decrease in cell growth, the catalytic activity of PKM2 is modulated allosterically by critical metabolic intermediates ${ }^{16-18}$ and post-translationally in response to growth factor signalling and reactive oxygen species $(\text { ROS })^{10,19}$, providing a focal point for the integration of cellular signalling and redox status with glycolytic flux. Here we show that loss of PKM2 in ECs results in TCA cycle dysfunction, cell cycle arrest and the induction of viral mimicry by endogenous retroviral transcripts.

\section{Results}

Loss of endothelial PKM2 alters mitochondrial metabolism. The PKM gene is abundantly expressed in ECs (Supplementary Figure 1a), and RT-qPCR (Fig. 1a) and western blot (Fig. 1b) analyses show that PKM2 is the predominant isoform. To determine the function of endothelial PKM2, we first assessed the efficacy of validated siRNA duplexes targeting specifically PKM2 $\left(\mathrm{PKM}{ }^{2 \mathrm{KD}}\right)$ or both $\mathrm{PKM}$ splice isoforms $\left(\mathrm{PKM}^{\mathrm{KD}}\right)$ in primary human umbilical vein ECs (HUVECs) (Supplementary Figure 1$)^{20}$. Functionally, while neither $\mathrm{PKM} 2^{\mathrm{KD}}$ nor $\mathrm{PKM}^{\mathrm{KD}}$ significantly changed cellular energy charge (Fig. 1c), incorporation of [U$13 \mathrm{C}_{6}$ ]-glucose-derived carbon into $\mathrm{m}+3$ lactate (Fig. 1d) and extracellular acidification rate (ECAR) (Supplementary Figure 1g) were significantly reduced in both conditions. The reduction in labelled lactate was coupled to an increase in $\left[\mathrm{U}-13 \mathrm{C}_{6}\right]$-glucose labelling of citrate (Fig. 1e) and oxygen consumption rate (OCR) (Fig. 1f) in PKM2 ${ }^{\mathrm{KD}}$ ECs but not in $\mathrm{PKM}^{\mathrm{KD}}$ ECs, indicating that PKM isoform expression is a critical determinant of the fate of glucose-derived carbon in ECs. Intriguingly, analysis of steady-state levels of TCA cycle intermediates revealed a decrease in total $\alpha$ ketoglutarate $(a-K G)$, fumarate and malate in $\mathrm{PKM}_{2} \mathrm{KD}$ and $\mathrm{PKM}^{\mathrm{KD}} \mathrm{ECs}$, while aspartate levels were significantly increased only in PKM $2{ }^{\mathrm{KD}}$ ECs (Supplementary Figure $1 \mathrm{~h}$ ). Furthermore, [U-13C 6 ]-glucose labelling of $\alpha$-KG (Fig. $1 \mathrm{~g}$ ) was significantly reduced in $\mathrm{PKM} 2^{\mathrm{KD}}$ and $\mathrm{PKM}^{\mathrm{KD}}$ ECs, while aspartate labelling was increased only in $\mathrm{PKM} 2^{\mathrm{KD}}$ ECs (Fig. 1h), indicating that significant changes to TCA cycle metabolism occur in the absence of PKM2. Glycolysis is not the sole carbon source for the TCA cycle, which is also fuelled by branched chain amino acid (BCAA) oxidation, pyruvate anaplerosis, fatty acid oxidation (FAO) and glutamine metabolism 3,9 . We found that $\mathrm{PKM}_{2} \mathrm{KD}$, but not $\mathrm{PKM}^{\mathrm{KD}}$, led to a reduction in the ratio of keto-leucine to isoleucine (Fig. 1i) and [U-13 $\left.\mathrm{C}_{6}\right]$-glucose labelling of $\mathrm{m}+3$ alanine (Fig. $1 \mathrm{j}$ ), while an accumulation of long-chain acylcarnitines (Fig. 1k), and reduced $\mathrm{m}+3$ malate (Supplementary Figure 1i) were observed in both $\mathrm{PKM} 2 \mathrm{KD}$ and $\mathrm{PKM}{ }^{\mathrm{KD}}$ ECs. These data indicate that BCAA oxidation, pyruvate anaplerosis and $\mathrm{FAO}$ are impaired in $\mathrm{PKM} 2{ }_{2}^{\mathrm{KD}}$ ECs. Mitochondria are an important source of ROS in endothelial cells, where at low levels ROS perform critical signalling functions $^{21}$. We found that while the global ratio of GSSG/GSH (Supplementary Figure 1j) and cellular ROS levels (Supplementary Figure $1 \mathrm{k}$ ) were not changed in $\mathrm{PKM} 2 \mathrm{KD} E C s$, there was an increase in mitochondrial superoxide levels (Fig. 11). Furthermore, we did not observe changes in mitochondrial DNA content (Supplementary Figure 11). Collectively, these data show that loss of PKM2 alters mitochondrial metabolism and is associated with the disruption of multiple pathways that fuel TCA cycle metabolism and cell growth.

Loss of PKM2 impairs angiogenic sprouting. To understand the functional importance of PKM2 for angiogenic sprouting, we assessed EC migration and proliferation in primary cells, zebrafish and mice. In HUVECs, PKM $2^{\mathrm{KD}}$ led to a decrease in the number of 5-ethynyl-2'-deoxyuridine positive $\left(\mathrm{EdU}^{+}\right)$(Fig. 2a) and phospho-Histone $\mathrm{H} 3$ positive $\left(\mathrm{pHH}_{3}{ }^{+}\right.$) (Supplementary Figure 2a) ECs, and cell cycle arrest in $\mathrm{G}_{0} / \mathrm{G}_{1}$ at $48 \mathrm{~h}$ after siRNA transfection (Supplementary Figure $2 \mathrm{~b}$ ), resulting in a significant reduction in relative EC number (Fig. 2b). We also observed a decrease in EC proliferation using ML265, a small-molecule activator of PKM2 tetramerization that mimics the effects of PKM2 loss of function, providing further evidence that modulation of PKM2 activity can inhibit EC proliferation (Supplementary Figure 2c). Furthermore, PKM2 ${ }^{\mathrm{KD}}$ significantly impaired wound closure in an in vitro scratch wound assay, indicating that PKM2 may also play an important role in EC migration (Fig. 2c). To assess the in vivo relevance of these findings, we generated mutants in the zebrafish orthologues, pkma and pkmb (Supplementary Figure $2 \mathrm{~d}-\mathrm{g}$ ). The zebrafish $p k m a$ gene displays a similar genomic organization as its mammalian orthologues (Supplementary Figure 2d), and is alternatively spliced into exon 9 or 10 to give rise to mRNAs that we have designated as pkmal and pkma2. In contrast, in zebrafish and other teleosts, the pkmb gene contains 11 exons and is not subject to alternative splicing (Supplementary Figure 2d). Clustering analysis based on a 56 amino acid sequence with homology to the amino acids encoded by exon 9 or 10 of the human PKM gene (Supplementary Figure $2 \mathrm{e}$ ), indicates that $p k m b$ is an orthologue of mammalian PKM2, not PKM1. Confocal analyses of wild-type, maternal zygotic pkma2 (pkma2 ${ }^{m z}$ ) and $p k m a 2^{m z} / p k m b^{-1-}$ mutant embryos revealed defects in the formation of the intersegmental vessels (ISVs) (Fig. 2d). Live imaging demonstrated impaired proliferation and migration of ECs in the ISVs of $p k m a 2^{m z} / \mathrm{pkmb}^{-1-}$ mutants (Supplementary Movie 1). Quantification of incomplete ISVs at $48 \mathrm{~h}$ post fertilization (hpf) reveals a significant increase in the number of ISVs that fail to make connections with their neighbours in $p k m a 2^{m z} / p m b^{-1-}$ mutants (Fig. 2e), indicating that $\mathrm{Pkm} 2$ regulates blood vessel growth in vivo.

To interrogate the endothelial cell autonomous requirement for PKM2 during vessel growth, we conditionally deleted exon 10 of the $P \mathrm{~km}$ gene (Supplementary Figure 3a, b) in endothelial cells of mice and analysed angiogenesis in the postnatal retina. To this 
a

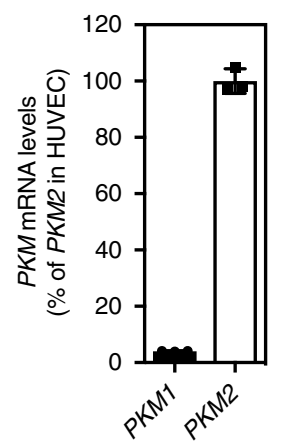

b

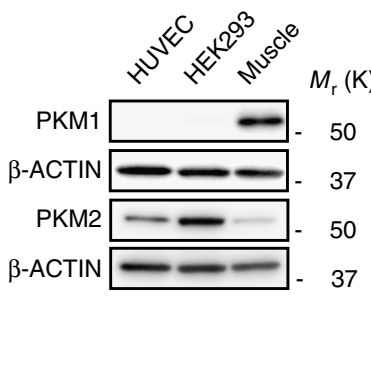

C

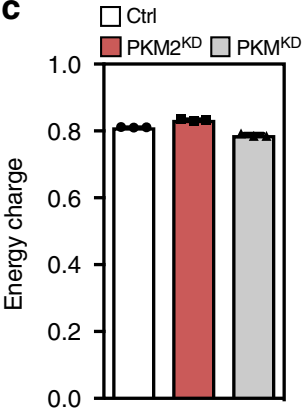

d

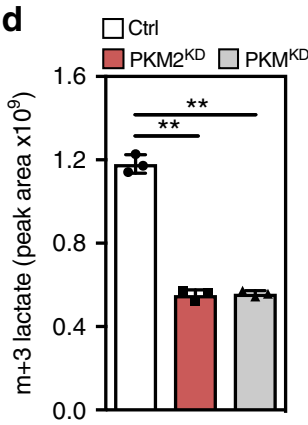

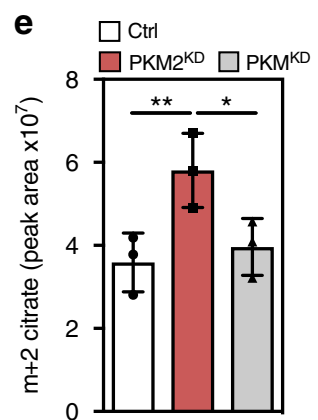

f

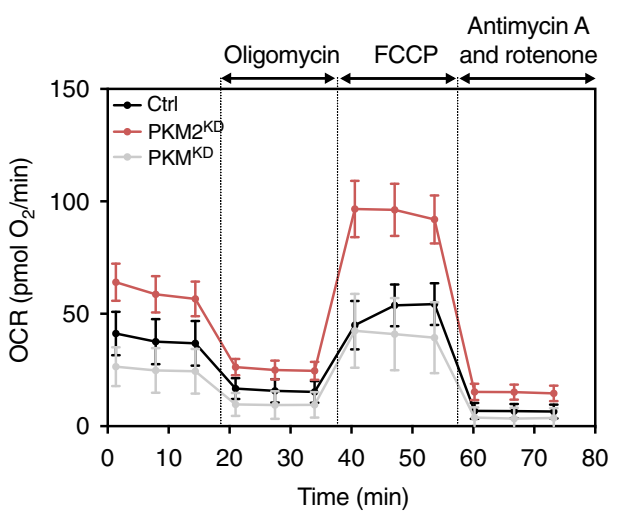

g

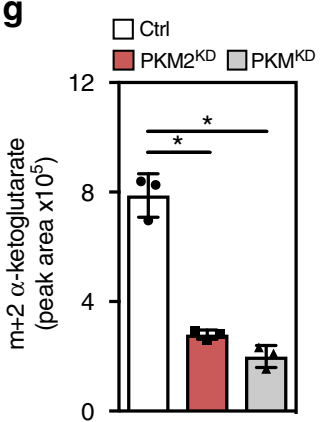

h
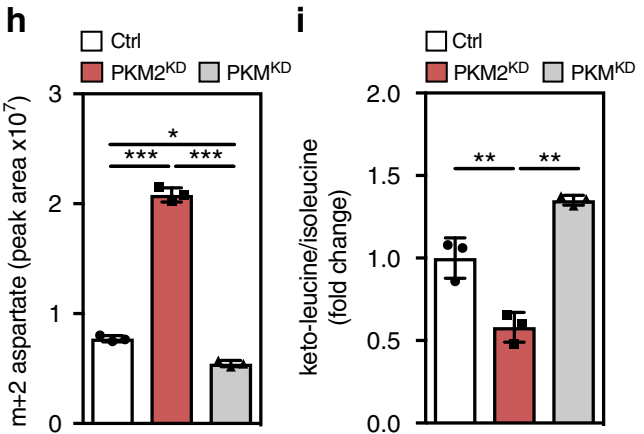

j

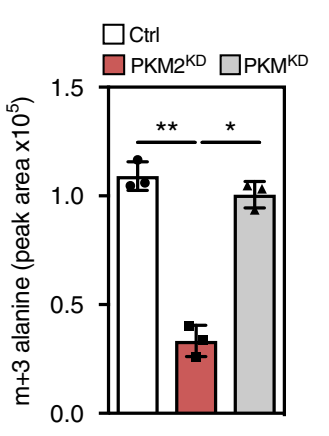

k

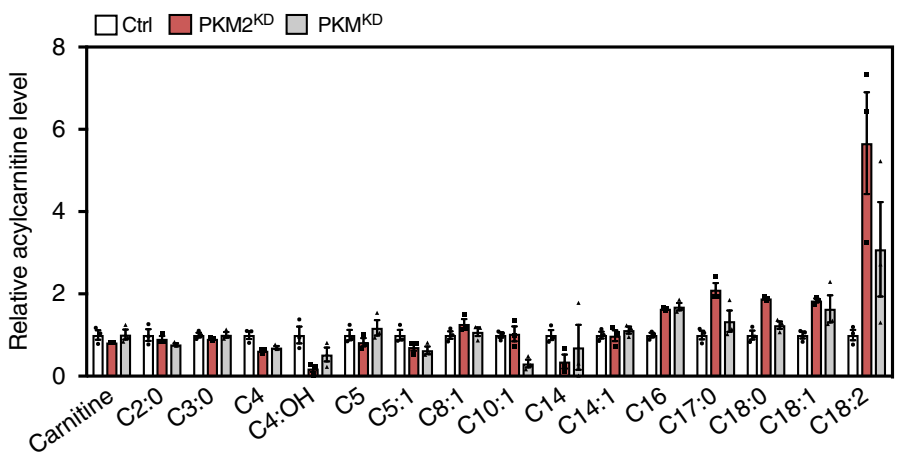

I

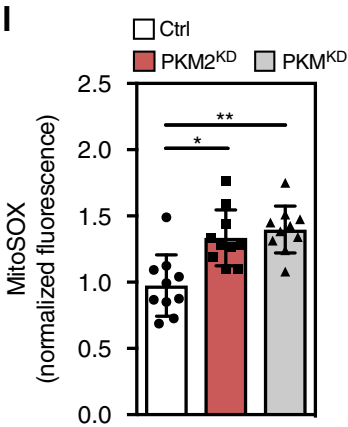

Fig. 1 Loss of endothelial PKM2 leads to mitochondrial dysfunction. a RT-qPCR analysis of PKM mRNA expression showing that PKM2 is more abundantly expressed in HUVECs $(n=3)$. b Western blot analysis of PKM1 and PKM2 expression in HUVECs, HEK293 and mouse skeletal muscle. c Relative energy charge $([\mathrm{ATP}]+1 / 2[\mathrm{ADP}] /[\mathrm{ATP}]+[\mathrm{ADP}]+[\mathrm{AMP}])$ in control, PKM2 ${ }^{\mathrm{KD}}$ and PKMKD $\mathrm{ECs}(n=3)$. Incorporation of $\left[\mathrm{U}-13 \mathrm{C}_{6}\right]$-glucose into $\mathrm{m}+3$ lactate (d) and $\mathrm{m}+2$ citrate $(\mathbf{e})$ in control, $\mathrm{PKM} 2^{\mathrm{KD}}$ and PKM ${ }^{\mathrm{KD}} \mathrm{ECs}(n=3)$. $\mathbf{f}$ Oxygen consumption rate (OCR) in control, PKM2 ${ }^{\mathrm{KD}}$ and PKM ${ }^{\mathrm{KD}} \mathrm{ECs}$ under basal conditions and in response to oligomycin, fluoro-carbonyl cyanide phenyl-hydrazone (FCCP) and antimycin A/rotenone $(n=6$, data represent mean $\pm s$. d.). $\mathbf{g}$ Incorporation of $\left[\mathrm{U}-13 \mathrm{C}_{6}\right]$-glucose into $\mathrm{m}+2 \alpha$-ketoglutarate in control, PKM2 ${ }^{\mathrm{KD}}$ and PKM ${ }^{\mathrm{KD}} \mathrm{ECs}(n=3)$. $\mathbf{h}$ Incorporation of $[\mathrm{U}$-13C 6 -glucose into $\mathrm{m}$ +2 aspartate in control, PKM2 ${ }^{\mathrm{KD}}$ and PKM ${ }^{\mathrm{KD}} \mathrm{ECs}(n=3)$. i Ratio of keto-leucine/isoleucine in control, PKM2 ${ }^{\mathrm{KD}}$ and PKM ${ }^{\mathrm{KD}}$ ECs. $\mathbf{j}$ Incorporation of $[\mathrm{U}$ $13 \mathrm{C}_{6}$ ]-glucose into $\mathrm{m}+3$ alanine in control, $\mathrm{PKM} 2^{\mathrm{KD}}$ and $\mathrm{PKM}{ }^{\mathrm{KD}} \mathrm{ECs}(n=3)$. $\mathbf{k}$ Relative levels of different acylcarnitine species in control, PKM2 ${ }^{\mathrm{KD}}$ and PKM ${ }^{K D}$ ECs $(n=3)$. I Normalized MitoSOX fluorescence intensity in control, PKM $2^{K D}$ and PKM ${ }^{K D}$ ECs. a, c-I Data represent means $\pm s . d$., $m+n$ represents the mass isotopomers for individual metabolites ( ${ }^{\star \star \star} P<0.001,{ }^{\star \star} P<0.01,{ }^{\star} P<0.05$ by one-way analysis of variance (ANOVA) followed by Tukey's HSD test)

end, we intercrossed $P k m 2^{f l o x}$ mice with $P d g f b-C r e^{E R T 2}$ mice (referred to hereafter as $P k m 2^{i E C-K O}$ ) and induced recombination by 4-hydroxy-tamoxifen (4-OHT) injection from postnatal day (P) 1-3. Staining of retinas for PECAM (marking EC membranes) and ERG (marking EC nuclei) at P7 revealed reduced outgrowth of a hypo-cellular vascular plexus in $P k m 2^{i E C-K O}$ mice (Fig. 3a, b). To determine the cause of this phenotype, we analysed the morphology and growth of vessels at the angiogenic front in PECAM, ERG and EdU-stained retinas (Fig. 3c). Quantification revealed a significant reduction in EC number, proliferation, vessel area and branching in $P k m 2^{i E C-K O}$ mice (Fig. 3d). In contrast to the vascular defects at the angiogenic front, the central plexus of the retina was less affected by PKM2 inactivation using the Pdgfb-Cre $e^{E R T 2}$ (Supplementary Figure $3 \mathrm{c}$ ). While endothelial cellularity was significantly reduced, vessel area and branch point density were similar to controls (Supplementary Figure 3e). These findings are consistent with the enrichment of PKM2 in angiogenic ECs ${ }^{22}$ and suggest that PKM2 signalling is particularly important for vessel sprouting at the growing angiogenic front. To assess the possible contribution of vessel 
a

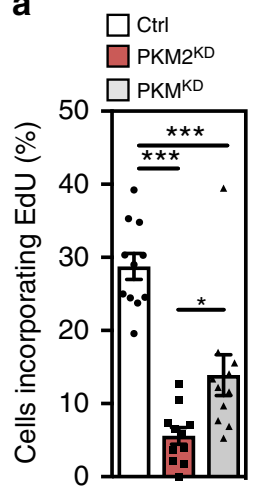

b

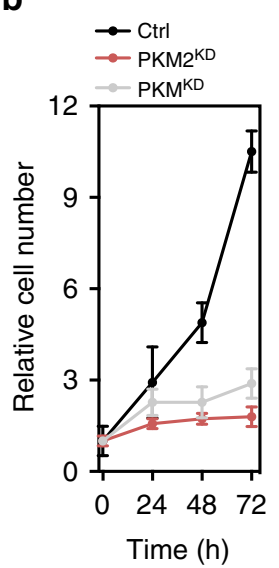

C

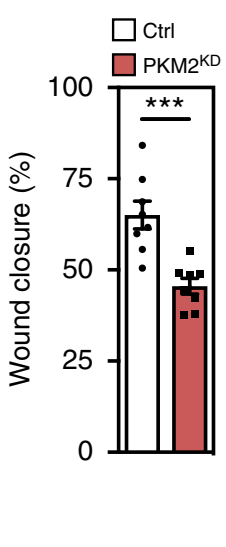

d

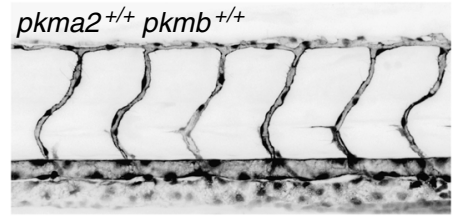

pkma2 ${ }^{M Z} \mathrm{pkmb}^{-/-}$

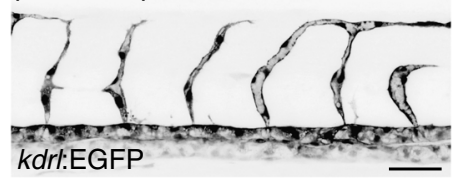

e

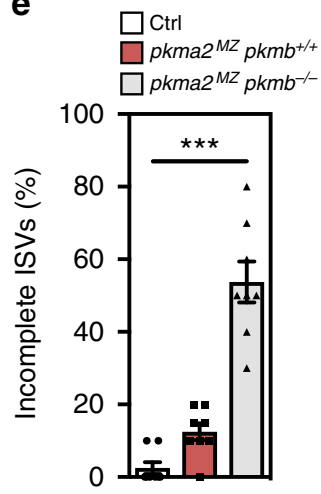

Fig. 2 Loss of PKM2 leads to angiogenic sprouting defects. a EdU-positive cell numbers in control, PKM2 ${ }^{K D}$ and PKM ${ }^{K D}$ ECs ( $\left.n=11\right)$. b Relative cell numbers at the indicated time points in control, PKM $2^{\mathrm{KD}}$ and PKMKD ECs $(n=6)$. c Scratch wound assay in control and PKM2 $2^{\mathrm{KD}} \mathrm{ECs}(n=8)$.

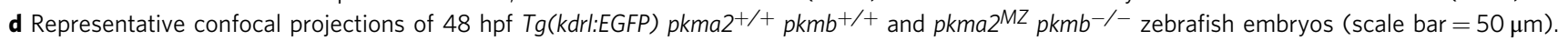
e Percentage of intersegmental vessels (ISVs) that have failed to connect with neighbouring ISVs in 48 hpf zebrafish embryos ( $n=8$ ). Data in a-c and e represent means \pm s.e.m. ( ${ }^{\star \star \star} P<0.001$ by one-way analysis of variance (ANOVA) followed by Tukey's HSD test (a, e) or two-tailed Student's $t$ test (c))

a

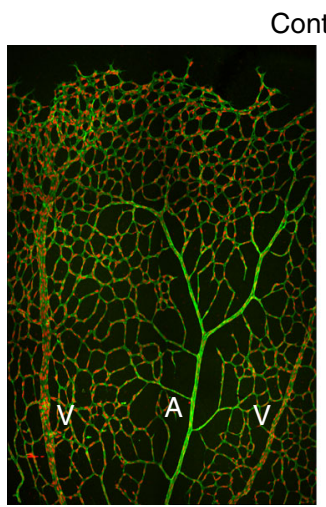

PECAM ERG

C

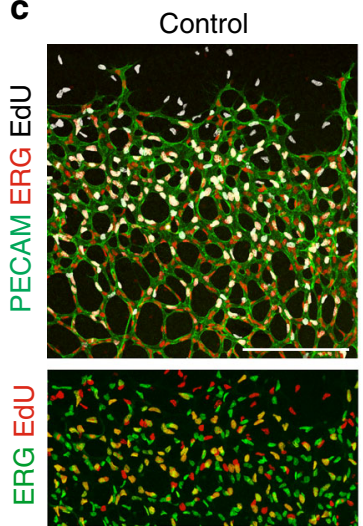

Control

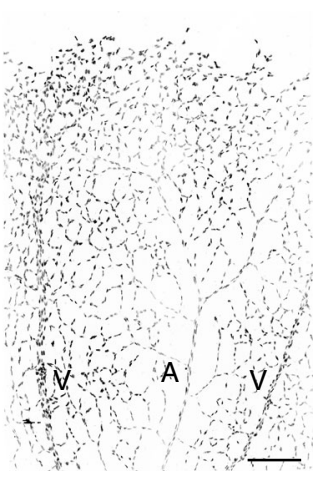

ERG

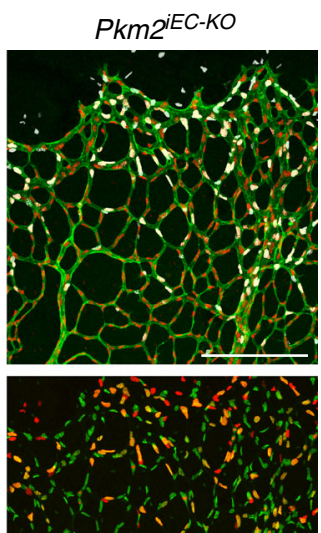

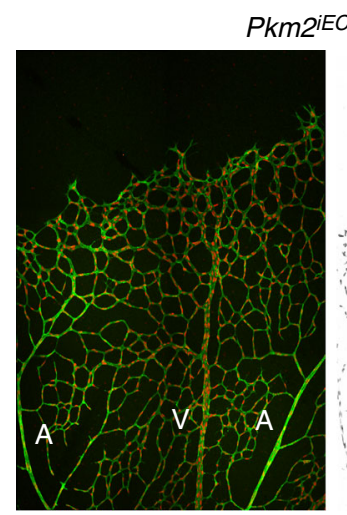

PECAM ERG

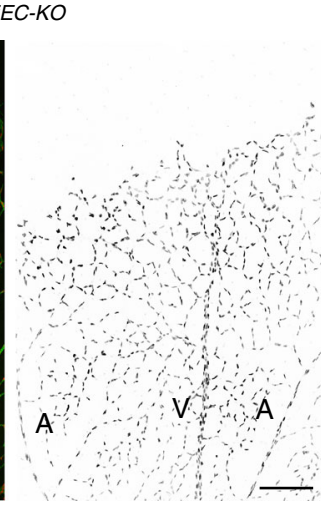

ERG b

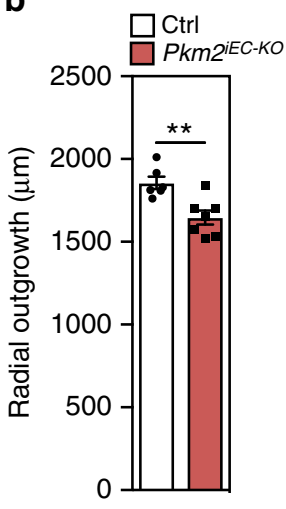

d

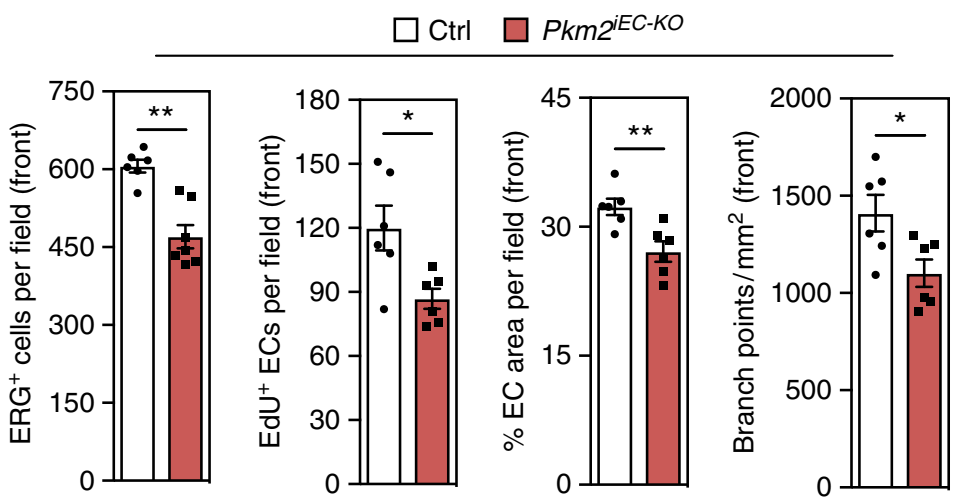

Fig. 3 Acute deletion of $P k m 2$ in mouse endothelial cells leads to angiogenic sprouting defects. a Representative confocal projections of PECAM, ERG and EdU immunostained retinas from control and $P k m 2^{i E C-K O}$ mice (A, Artery; $V$, Vein). b Radial outgrowth in control and $P k m 2^{i E C-K O}$ mice $(n=6)$. c Representative confocal projections of angiogenic front vessels in PECAM, ERG and EdU-stained retinas from control and Pkm2 $2^{i E C-K O}$ mice. $\mathbf{d}$ ERG ${ }^{+}$ECs, $\mathrm{EdU}^{+} \mathrm{ECs}$, EC area per field and branch point density at the angiogenic front of control and $P k m 2^{i E C-K O}$ mice $(n=6)$. All analyses in the mouse retina were performed on postnatal day 7. Data in $\mathbf{b}$ and $\mathbf{d}$ represent means \pm s.e.m. ( ${ }^{\star \star} P<0.01,{ }^{\star} P<0.05$ by two-tailed Student's $t$ test). Scale bars in $\mathbf{a}$ and $\mathbf{c}=$ $200 \mu \mathrm{m}$ 
a

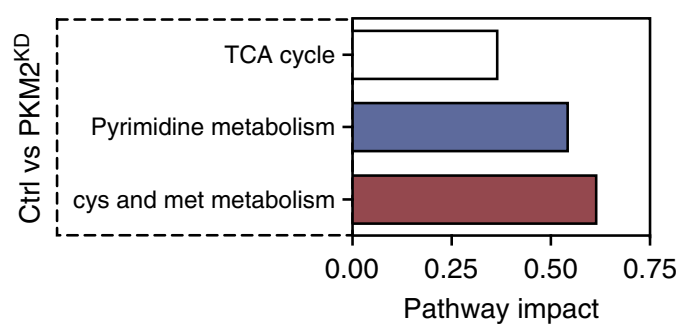

b

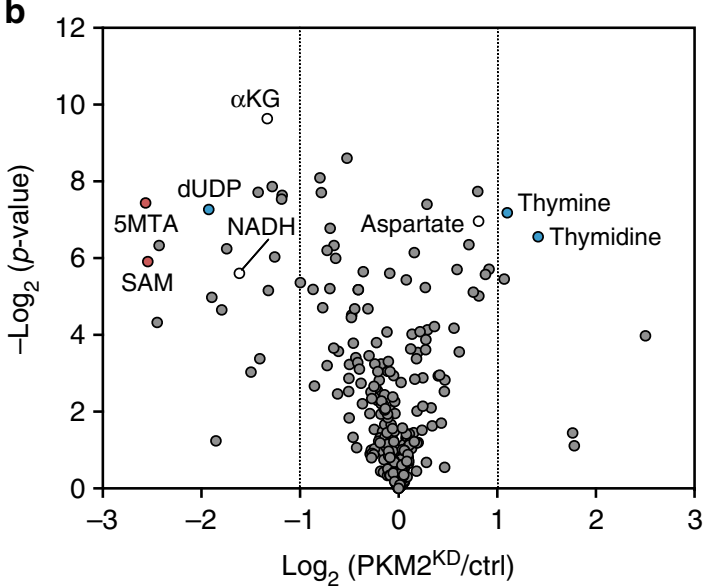

C
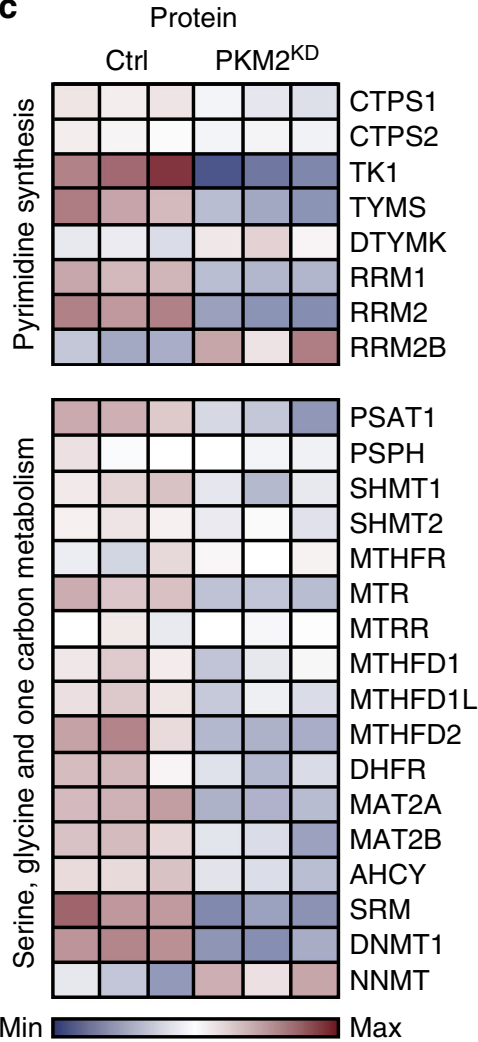

Fig. 4 Loss of PKM2 leads to coordinated metabolic and protein expression changes. a Analysis of steady-state metabolite levels in control vs PKM2KD ECs identifies the TCA cycle, pyrimidine metabolism and cysteine and methionine metabolism as significantly regulated pathways. $\mathbf{b}$ Metabolomics profile of control vs PKM2 ${ }^{\mathrm{KD}}$ ECs ( $\log _{2}$ (fold change) vs $-\log _{2}$ ( $p$ value), two-tailed Student's $t$ test, $n=3$ ). c Heat map of protein expression for key enzymes in the pyrimidine synthesis and serine, glycine, one-carbon metabolic networks in control vs PKM2 ${ }^{\mathrm{KD}} \mathrm{ECs}$

regression, we quantified the number of empty collagen $\mathrm{IV}^{+}$ sleeves and observed no significant difference between control and $P k m 2^{i E C-K O}$ mice (Supplementary Figure $3 \mathrm{~d}, \mathrm{e}$ ). Taken together, these data suggest that PKM2 is a crucial regulator of endothelial migration and proliferation, whose inactivation compromises vascular expansion.

Coordinated metabolic and protein changes following PKM2 loss. To gain further insight into the global metabolic changes associated with loss of endothelial PKM2, we performed pathway analyses of steady-state metabolites and identified pathways linked to mitochondrial metabolism ${ }^{23,24}$, including TCA cycle, pyrimidine synthesis and cysteine and methionine metabolism (Fig. 4a, specific metabolites highlighted in Fig. 4b). Deletion of PKM2 in primary mouse embryonic fibroblasts (MEFs) leads to impaired pyrimidine synthesis that does not correlate with gene expression changes ${ }^{25}$. To determine if loss of PKM2 is associated with gene expression changes in ECs, we performed a proteomics analysis that revealed changes to the expression of a number of key enzymes in the pyrimidine synthesis, and serine, glycine and one-carbon metabolism (SGOC) pathways (Fig. 4c and Supplementary Figure 4a). These data indicate that the metabolic changes observed in $\mathrm{PKM} 2^{\mathrm{KD}}$ ECs (Fig. $4 \mathrm{a}, \mathrm{b}$ ) correlate with protein-level changes in the key enzymes controlling these pathways, leading us to hypothesize that there may be a coordinated transcriptional response to the loss of PKM2 in ECs.

RELB-P53 suppresses endothelial growth in the absence of PKM2. Further analyses revealed that components of the NF- $\kappa B$ and TP53 transcription factor pathways, which have previously been linked with the regulation of metabolism ${ }^{26-29}$, are upregulated in PKM2 ${ }^{\mathrm{KD}}$ ECs (Supplementary Figure $5 \mathrm{a}, \mathrm{b}$ ). We thus set out to test if these pathways regulate EC growth in the absence of PKM2. Co-transfection of siRNA duplexes targeting PKM2 and REL, RELA or RELB, revealed that silencing RELB significantly rescued the number of EdU ${ }^{+}$(Fig. 5a and Supplementary Figure 5c) and $\mathrm{pHH}^{+}$(Fig. 5a) ECs, indicating that RELB mediates the cell cycle arrest observed in the absence of PKM2. Western blot analysis of control, $\mathrm{RELB}^{\mathrm{KD}}, \mathrm{PKM} 2^{\mathrm{KD}}$ and $\mathrm{PKM} 2^{\mathrm{KD}} / \mathrm{RELB}^{\mathrm{KD}}$ ECs revealed that RELB expression was increased in the absence of PKM2 and acts to induce the expression of P53 and its downstream target P21 (Fig. 5b).

Given the established role of the P53-P21 pathway in the regulation of cell cycle progression ${ }^{30}$, we hypothesized that RELBmediated induction of P53-P21 might be the cause of the cell cycle arrest observed in PKM2 ${ }^{\mathrm{KD}}$ ECs. Quantification of EdU and phospho-Histone H3 (Fig. 5c) positive ECs, and total cell numbers (Supplementary Figure 5d) revealed a significant rescue of the cell cycle arrest observed in $\mathrm{PKM} 2{ }^{\mathrm{KD}}$ ECs following P53 silencing. Furthermore, western blot analysis showed that the increase in P53 and its transcriptional target P21 in PKM $2^{\mathrm{KD}} \mathrm{ECs}$ was abolished by co-transfecting siRNA duplexes targeting P53, while RELB expression was unchanged (Fig. 5d). These data show that RELB acts upstream of P53-P21 to induce cell cycle arrest in the absence of PKM2. In addition to its well-documented tumour suppressor functions $s^{30}$, there is an increasing appreciation of the importance of P53 in the regulation of metabolism in response to various types of cellular stress ${ }^{26}$. In tumour cells deprived of exogenous serine, P53 promotes glutathione synthesis at the expense of nucleotides to promote cell survival ${ }^{31}$. To identify potential transcriptional and metabolic changes controlled by 
a

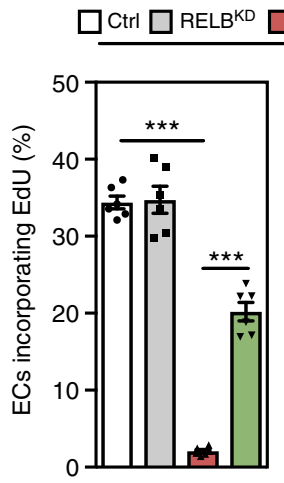

b

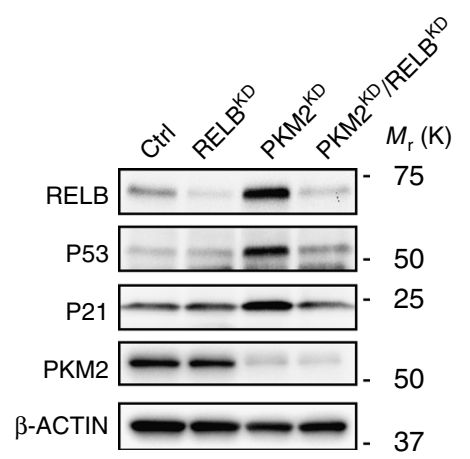

C $\quad \square$ Ctrl $\square$ P53 $3^{\mathrm{KD}} \quad \square \mathrm{PKM}^{\mathrm{KD}} \square \mathrm{PKM}^{\mathrm{KD} / \mathrm{P} 53^{\mathrm{KD}}}$

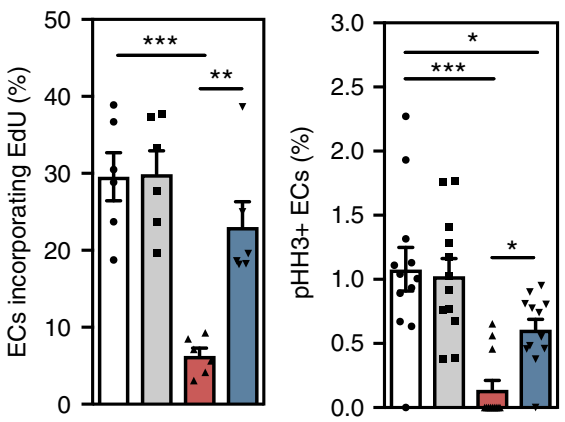

d

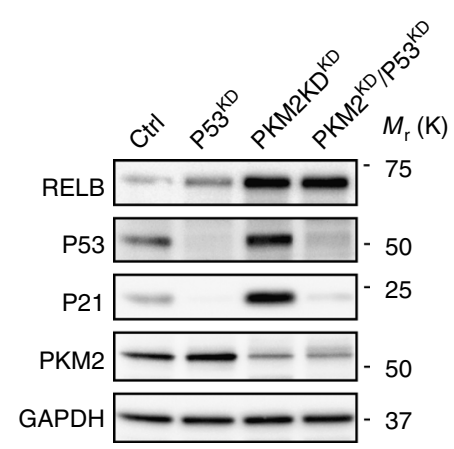

e $\square \mathrm{Ctrl} \quad \square \mathrm{P} 53^{\mathrm{KD}} \quad \square \mathrm{PKM} 2^{\mathrm{KD}} \quad \square \mathrm{PKM} 2^{\mathrm{KD}} / \mathrm{P} 53^{\mathrm{KD}}$
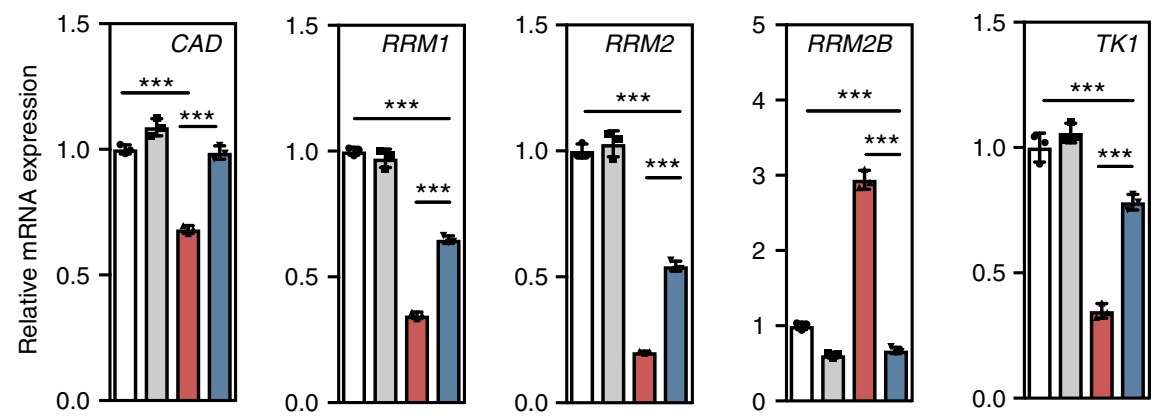

f $\square \mathrm{Ctrl} \quad \square \mathrm{P} 53^{\mathrm{KD}} \quad \square \mathrm{PKM} 2^{\mathrm{KD}} \quad \square \mathrm{PKM} 2^{\mathrm{KD}} / \mathrm{P} 53^{\mathrm{KD}}$

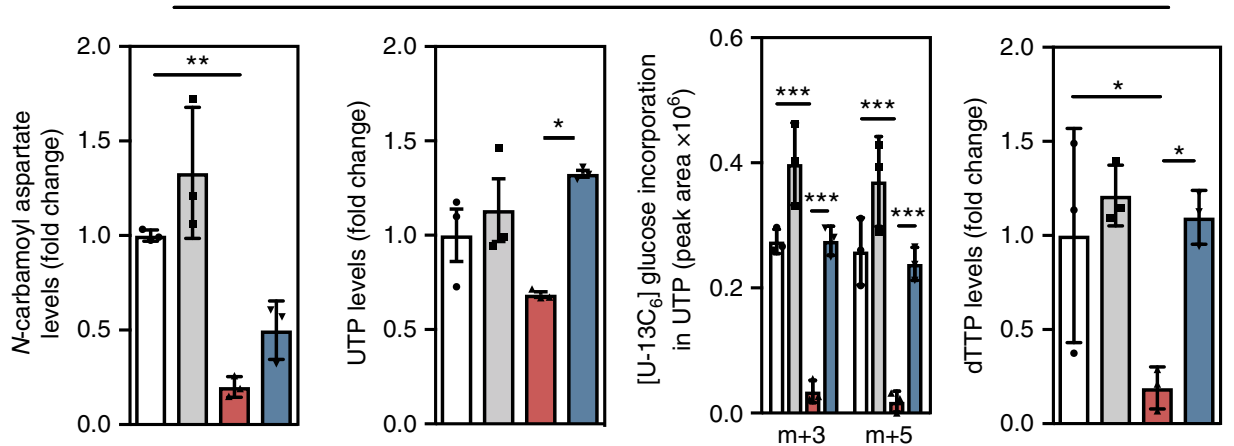

Fig. 5 RELB-P53 suppresses endothelial growth in the absence of PKM2. a EdU and phospho-Histone H3 positive cell numbers in control, RELBKD, PKM2KD

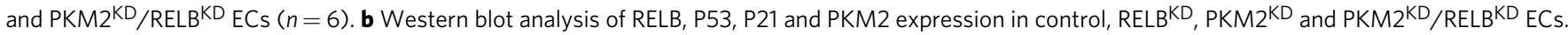
c EdU and phospho-Histone $\mathrm{H} 3$ positive cell numbers in control, P53 KD, PKM2 KD and PKM2 ${ }^{\mathrm{KD}} / \mathrm{P} 53^{\mathrm{KD}} \mathrm{ECs}(n=12)$. d Western blot analysis of RELB, P53, $\mathrm{P} 21$ and $\mathrm{PKM} 2$ expression in control, P53 KD, PKM2 ${ }^{\mathrm{KD}}$ and PKM2 KD/P53KD ECs. e mRNA expression levels of key enzymes for pyrimidine synthesis in control, P53 $3^{K D}, P K M 2^{K D}$ and PKM2 ${ }^{K D} / P 53^{K D}$ ECs (RNA-seq analysis, $n=3$ ). f Relative $N$-carbamoyl aspartate levels, UTP levels, incorporation of [U$13 \mathrm{C}_{6}$ ]-glucose into $\mathrm{m}+3$ and $\mathrm{m}+5 \mathrm{UTP}$, and relative dTTP levels in control, P53 ${ }^{\mathrm{KD}}, \mathrm{PKM} 2^{\mathrm{KD}}$ and PKM2 ${ }^{\mathrm{KD}} / \mathrm{P} 53^{\mathrm{KD}} \mathrm{ECs}(n=3)$. a, $\mathbf{c}$ Data represent means \pm s.e.m., e, $\mathbf{f}$ data represent means \pm s.d., $m+n$ represents the mass isotopomers for individual metabolites $\left({ }^{\star \star \star} P<0.001,{ }^{\star \star} P<0.01,{ }^{\star} P<0.05\right.$ by one-way analysis of variance (ANOVA) followed by Tukey's HSD test) 
P53, we performed RNA-seq and metabolomics analyses in control, $\mathrm{P} 53^{\mathrm{KD}}, \mathrm{PKM} 2^{\mathrm{KD}}$ and $\mathrm{PKM} 2^{\mathrm{KD}} / \mathrm{P} 53^{\mathrm{KD}}$ ECs. Analysis of the transcriptional response (Supplementary Figure 5e) revealed that P53 regulates the expression of key enzymes in the pyrimidine synthesis pathway (Fig. 5e). Volcano plots displaying metabolite profiles show that the relative levels of a number of metabolites in the pyrimidine synthesis pathway are reduced following $\mathrm{PKM} 2{ }^{\mathrm{KD}}$ (Supplementary Figure $5 \mathrm{f}$ ), and that silencing of P53 leads to a relative normalization of the levels of these metabolites (Supplementary Figure 5g). Comprehensive analysis of nucleotide levels revealed that $\mathrm{PKM} 2{ }^{\mathrm{KD}}$ reduced the relative levels of $\mathrm{N}$-carbamoyl aspartate, UTP and dTTP, and impaired incorporation of glucose-derived carbon into UTP, while silencing of P53 increased the levels of these metabolites (Fig. 5f). Collectively, these data indicate that a P53-dependent transcriptional programme controls the reduction in pyrimidine nucleotide levels in ECs lacking PKM2.

Loss of PKM2 impairs methylation capacity and DNA methylation. In addition to changes in the pyrimidine synthesis pathway, loss of PKM2 also led to significant changes in the steady-state levels of the one-carbon metabolite sadenosylmethionine (SAM) (Fig. 4b), which were coupled to reduced expression of enzymes in the SGOC network (Fig. 4c). As the primary cellular methyl group donor, the abundance of SAM determines the methylation status of a broad range of substrates, including DNA and histones ${ }^{32}$. SAM is an intermediate metabolite in a wider metabolic network that encompasses the folate and methionine cycles, termed one-carbon metabolism ${ }^{24}$, and is synthesized from ATP and methionine, by methionine adenosyltransferases (MAT). MAT1A expression is restricted to the liver, while MAT2A and MAT2B are expressed in non-hepatic tissues and their expression levels have been shown to positively correlate with DNA methylation ${ }^{33}$. Western blot analysis of control, $\mathrm{P} 53^{\mathrm{KD}}, \mathrm{PKM} 2^{\mathrm{KD}}$ and $\mathrm{PKM} 2^{\mathrm{KD}} / \mathrm{P} 53^{\mathrm{KD}}$ ECs revealed a $\mathrm{P} 53$-independent reduction in the expression of MAT2A in the absence of PKM2 (Fig. 6a). Metabolomic analyses of control, $\mathrm{P} 53^{\mathrm{KD}}, \mathrm{PKM} 2^{\mathrm{KD}}$ and $\mathrm{PKM} 2^{\mathrm{KD}} / \mathrm{P} 53^{\mathrm{KD}}$ ECs showed a significant reduction in the steady-state levels of SAM (Fig. 6b), with no concomitant decrease in S-adenosylhomocysteine (SAH) in $\mathrm{PKM} 2^{\mathrm{KD}}$ and $\mathrm{PKM} 2^{\mathrm{KD}} / \mathrm{P} 53^{\mathrm{KD}}$ ECs (Fig. 6b), leading to a P53independent drop in the ratio of SAM:SAH (Fig. 6b). These changes were associated with a reduction in the SAM-derived metabolite S-methyl-5-thioadenosine (5MTA) in PKM2 ${ }^{\mathrm{KD}}$ and $\mathrm{PKM} 2^{\mathrm{KD}} / \mathrm{P} 53^{\mathrm{KD}} \mathrm{ECs}$, with no change in methionine levels or GSH/GSSG ratio (Supplementary Figure 6a). Altogether, these data indicate that loss of PKM2 leads to reduced levels of SAM, in a manner that is independent of cell cycle arrest or regulation by P53. A reduction in the SAM:SAH ratio has been linked to a decrease in cellular methylation capacity ${ }^{34}$, and as a consequence, we identified a significant reduction in global DNA methylation, as assessed by measuring the levels of methylated cytosine in control and PKM2 ${ }^{\mathrm{KD}}$ ECs (Fig. 6c).

Loss of PKM2 leads to endogenous retrovirus expression. Methylation of DNA controls a number of biological processes that include promoter inactivation, $\mathrm{X}$-chromosome silencing and the silencing of transposable elements ${ }^{35}$. Retrotransposons make up close to $45 \%$ of the human genome $\mathrm{e}^{36}$ and consist of two classes, long terminal repeat (LTR) and non-LTR transposons ${ }^{37}$. Derepression of these elements is associated with activation of the endogenous antiviral defence pathway ${ }^{37}$. In response to an increase of cytosolic viral RNA, a signalling cascade is triggered that begins with recognition by cytosolic sensors, activation of interferon regulatory factor (IRF)-NF- $\mathrm{BB}$ signalling and subsequent induction of interferon-stimulated gene (ISG) expression ${ }^{38}$. Intriguingly, global knockout of $P \mathrm{~km} 2$ in mouse was recently shown to increase systemic inflammation ${ }^{14}$, and $P k m 2^{-/-}$MEFs display increased expression of the ISGs Ifi44 and Ifih $1^{25}$, leading us to hypothesize that loss of PKM2 may lead to the activation of innate immune signalling as a consequence of reduced DNA methylation. Analysis of our proteomics data set revealed changes in the expression of genes associated with antiviral defence (Fig. 6d and Supplementary Figure 6b). Furthermore, mRNA levels of a number of ISGs were increased in PKM2 ${ }^{\mathrm{KD}}$ ECs (Supplementary Figure 6c), and analysis of published microarray data ${ }^{25}$ revealed that ISGs are induced in $P \mathrm{~km} 2^{-/}$MEFs (Supplementary Figure 6d), indicating that loss of PKM2 can lead to the activation of an innate immune response in multiple cell types and species. DNA methyltransferase (DNMT) inhibition was recently shown to permit the expression of endogenous retroviral (ERV) elements, leading to the formation of cytosolic dsRNA and activation of innate antiviral signalling 39,40 . IFIH1 (MDA5) encodes a cytosolic pattern recognition receptor that recognizes double-stranded RNA (dsRNA) ${ }^{41}$, and its expression is upregulated in both PKM2 loss-of-function ECs (Supplementary Figure 6c) and MEFs (Supplementary Figure 6d). We therefore investigated if loss of PKM2 might lead to the accumulation of dsRNAs in ECs using the $\mathrm{J} 2$ antibody, and found a significant increase in dsRNA levels in PKM2 ${ }^{\mathrm{KD}}$ ECs (Fig. 6e, f). In addition, RT-qPCR analyses of control and PKM2 ${ }^{\mathrm{KD}} \mathrm{ECs}$ revealed an increase in the expression of a subclass of ERVs (Fig. $6 \mathrm{~g}$ ), which are induced in response to DNMT inhibition ${ }^{39}$. To understand if increased ERV expression in PKM2 ${ }^{\mathrm{KD}}$ ECs was linked to changes in DNA methylation, we performed site-specific restriction digestion of PCR-amplified bisulfite-converted DNA at the MLT1B and MER4D loci. These analyses revealed a mild reduction in DNA methylation at the $M L T 1 B$ and MER4D loci in PKM2 ${ }^{\mathrm{KD}}$ ECs (Fig. 6h), the extent of which was likely limited by the cell cycle arrest observed following loss of PKM2. Histone H3 lysine 9 trimethylation (H3K9me3), which has been shown to contribute to ERV repression in embryonic stem cells ${ }^{42}$, was not changed in PKM2 ${ }^{\mathrm{KD}}$ ECs (Supplementary Figure 7a). Collectively, our data indicate that loss of PKM2 leads to reduced DNA methylation, which triggers viral mimicry induced by expression of endogenous retroviral transcripts.

ERV expression levels are limited by RELB. P53 has been shown to limit the expression of retrotransposons in MEFs treated with DNMT inhibitors ${ }^{43}$, and the NF- $\kappa \mathrm{B}$ transcription factor family is known to induce the expression of ISGs, forming a critical component of the innate immune response to viral infection ${ }^{44}$. Given the increased expression of both P53 and RELB in the absence of PKM2, we hypothesized that these transcription factors may regulate ISG and/or ERV expression in ECs. Loss of P53 did not significantly alter ISG expression (Supplementary Figure 7b), ERV expression (Supplementary Figure 7b) or DNA methylation (Supplementary Figure 7c) in PKM2 ${ }^{\mathrm{KD}}$ ECs. In contrast, RELB silencing led to a dramatic increase in ERV and ISG mRNA levels in both RELB ${ }^{\mathrm{KD}}$ and $\mathrm{PKM} 2 \mathrm{KD} / \mathrm{RELB}^{\mathrm{KD}} \mathrm{ECs}$ (Fig. 6i). Furthermore, analysis of previously published microarray data from $\mathrm{Relb}^{-/-}$mouse bone-marrow-derived dendritic cells revealed increased expression of numerous ISGs (Supplementary Figure $7 \mathrm{~d})^{45}$, indicating that RELB limits innate immune activation in multiple cell types. RELB is known to suppress transcription through the directed recruitment of DNA methyltransferases and chromatin remodelling factors ${ }^{46}$, leading us to hypothesize that RELB may limit ERV expression by regulating local chromatin composition. Analysis of the MLT1B and MER4D loci showed that $\mathrm{RELB}^{\mathrm{KD}}$ did not significantly alter DNA methylation (Supplementary Figure 7e), while H3K9me3 levels 
a

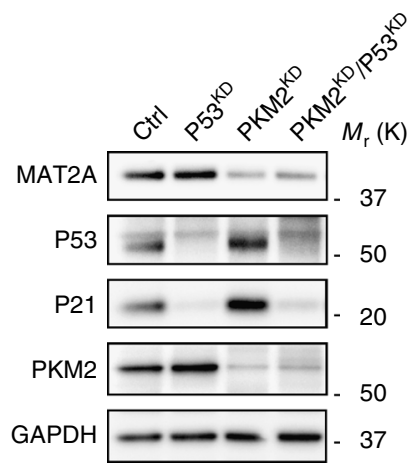

C

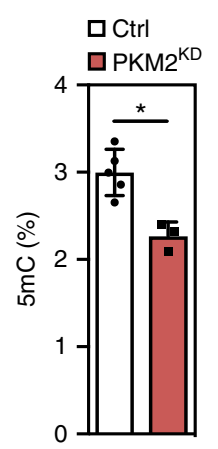

g

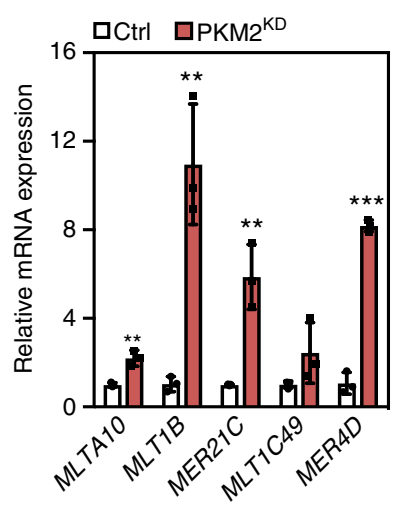

b

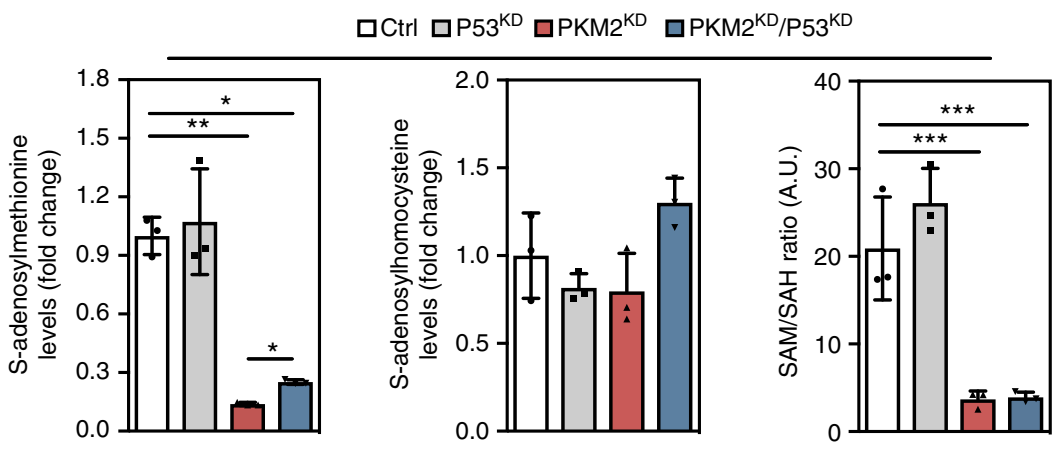

e

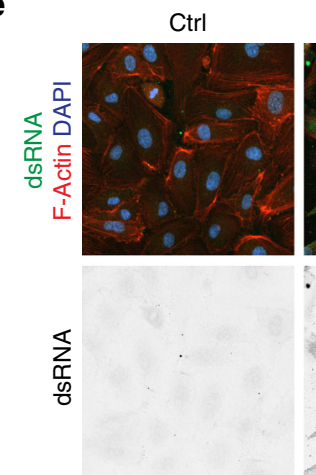

$\mathrm{PKM} 2^{\mathrm{KD}}$

f

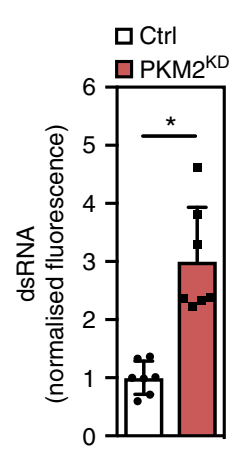

i

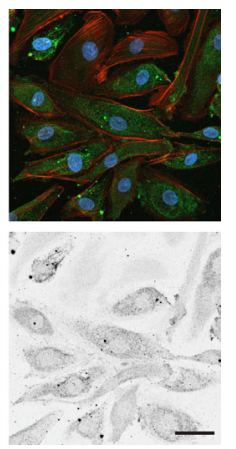

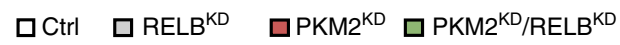

h

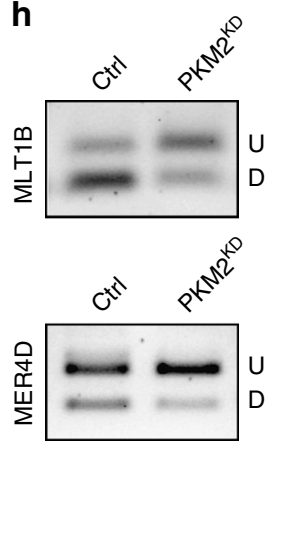

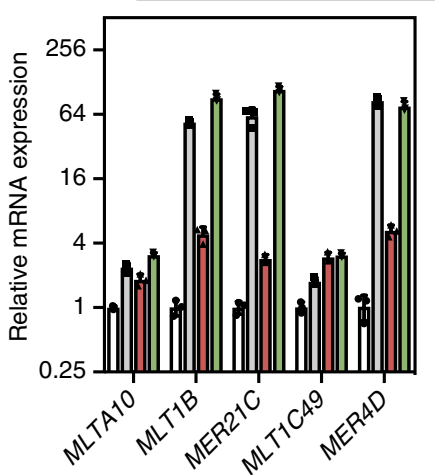

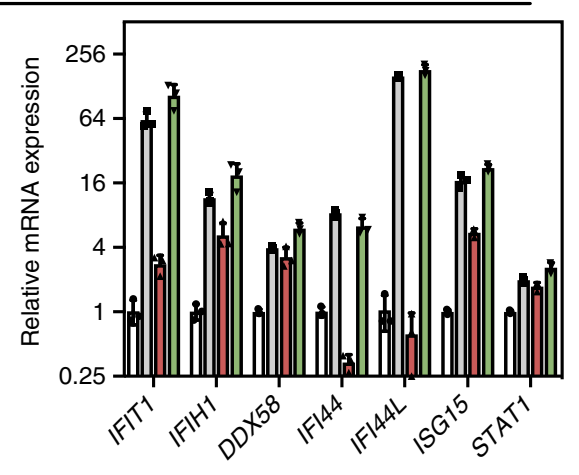

Fig. 6 Loss of PKM2 impairs methylation capacity, reduces DNA methylation and leads to the expression of endogenous retroviral elements. a Western blot analysis of MAT2A, P53, P21 and PKM2 in control, P53KD, PKM2KD and PKM2KD/P53KD ECs. b Relative levels of S-adenosylmethionine and Sadenosylhomocysteine and SAM/SAH ratio in control, P53 KD, PKM2 ${ }^{\mathrm{KD}}$ and PKM2 ${ }^{\mathrm{KD}} / \mathrm{P} 53^{\mathrm{KD}} \mathrm{ECs}(n=3)$. c Percentage 5 -methylcytosine $(5 \mathrm{mC})$ levels in control and PKM2 $2^{K D}$ ECs $(n=4)$. d Heat map of protein expression for components of the cellular response to viral infection in control and PKM2 ${ }^{K D}$ ECs. e Representative confocal projections of total double-stranded RNA (dsRNA) staining in control and PKM2 ${ }^{K D}$ ECs (scale bar $=20 \mu \mathrm{m}$ ). $\mathbf{f}$ Normalized fluorescence intensity for dsRNA staining in control and PKM2 ${ }^{\mathrm{KD}} \mathrm{ECs}(n=7)$. $\mathbf{g}$ Relative mRNA expression of the indicated endogenous retroviruses in control and PKM2KD ECs $(n=3)$. $\mathbf{h}$ Restriction digestion of bisulfite-treated DNA amplified from the MLT1B and MER4D genomic loci from control and PKM2 $2^{K D}$ ECs ( $U$, undigested/unmethylated DNA; D, digested/methylated DNA). i Relative mRNA expression of the indicated endogenous retroviruses and interferon-stimulated genes in control, RELBKD, PKM2 ${ }^{\mathrm{KD}}$ and PKM2 ${ }^{\mathrm{KD}} / \mathrm{RELBKD}$ ECs. b, $\mathbf{c}, \mathbf{f}, \mathbf{g}$, i Data represent means \pm s.d. $\left({ }^{\star \star *} P<0.001,{ }^{* \star} P<0.01,{ }^{*} P\right.$ $<0.05$ by one-way analysis of variance (ANOVA) followed by Tukey's HSD test)

were also unchanged (Supplementary Figure 7f). Collectively, these data show that RELB is a key regulator of the innate immune response in ECs, which acts in a DNA methylation and H3K9me3-independent manner to limit ERV expression (Supplementary Figure $7 \mathrm{~g}$ ).

\section{Discussion}

This study highlights the importance of PKM2 for growth and homoeostasis of the vascular endothelium and identifies molecular changes associated with its absence. PKM2 has been linked with anabolic cell growth in various experimental settings ${ }^{10,11,47}$, and its loss is associated with a PKM1-dependent impairment of pyrimidine synthesis and cell cycle arrest in $\mathrm{MEFs}^{25}$. In the present study, we observed distinct changes in metabolism in $\mathrm{PKM}^{\mathrm{KD}}$ and $\mathrm{PKM}^{\mathrm{KD}} \mathrm{ECs}$, indicating that the $\mathrm{PKM}$ isoforms differentially regulate EC metabolism. For example, oxygen consumption and $\left[\mathrm{U}-13 \mathrm{C}_{6}\right]$-glucose labelling of citrate were increased only in $\mathrm{PKM} 2{ }^{\mathrm{KD}}$ ECs, indicating that PKM1 may enhance the oxidative metabolism of glucose in mitochondria. In contrast, we observed reduced labelling of lactate and $\alpha-K G$, and increased mitochondrial superoxide levels in $\mathrm{PKM} 2{ }^{\mathrm{KD}}$ and 
$\mathrm{PKM}^{\mathrm{KD}}$ ECs, indicating that the levels of these metabolites are predominantly regulated by PKM2. Recent genetic analyses in mouse have demonstrated that chronic deletion of PKM2 does not impact cell proliferation, due to compensatory upregulation of the $P \mathrm{~km}$ splice isoform PKM1 ${ }^{14,48}$. Our genetic analysis of PKM2 function, using conditional deletion of PKM2 specifically in ECs, reveals that loss of PKM2 reduces EC proliferation in vivo and indicates that acute loss or transient inhibition of PKM2 may lead to fundamentally different effects on cell growth than chronic loss. Furthermore, we provide evidence that impaired nucleotide synthesis in cells lacking PKM2 can be regulated at the transcriptional level by RELB-P53 signalling. The NF-kB and P53 transcriptional networks are known to respond to a variety of cellular stress stimuli, and our data indicate that RELB-P53 signalling may play an important role in the response of the endothelium to metabolic dysfunction, by limiting cell growth.

There is an increasing appreciation of the important link between intermediary metabolism and chromatin structure and function 32,49 , with the availability of nutrients linked to modifications of $\mathrm{DNA}^{50}$ and histones ${ }^{51}$. One of the most striking metabolic changes that we observed in ECs lacking PKM2 was a reduction in the one-carbon metabolite, SAM, which is required for the methylation of a broad range of substrates, including cytosine residues in DNA. In yeast, the PKM2 orthologue Pyk1 was identified as a component of a complex named SESAME (serine-responsive SAM containing metabolic enzyme complex), that was shown to regulate chromatin modifications $s^{52}$. While in the present study we did not identify an orthologous mammalian SESAME complex, we observed protein-level changes in a number of key enzymes in the serine, glycine and one-carbon metabolic network. Decreased expression of the SAMsynthesizing enzyme MAT2A and reduced steady-state levels of SAM were independent of P53, indicating that this effect is not merely a consequence of cell cycle arrest. Taken together, these data indicate that loss or inhibition of PKM2 may lead to changes in chromatin structure and function.

As a consequence of DNA hypomethylation, we observed increased ERV, dsRNA and ISG levels in ECs lacking PKM2. The fold induction of ERV and ISG expression in PKM $2^{\mathrm{KD}}$ ECs was more limited than previously reported in HCT116 cells treated with DNMT inhibitors ${ }^{40}$. These differences may reflect the relatively minor changes in DNA methylation observed in $\mathrm{PKM} 22^{\mathrm{KD}}$ ECs, or result from cell-type-specific differences in ERV derepression, as previously described ${ }^{53}$. Examination of published microarray analyses of $P k m 2$ knockout $\mathrm{MEFs}^{25}$ shows increased expression of a number of ISGs, indicating that loss of PKM2 can trigger innate immune signalling in different cell types. In addition to its role in the maintenance of metabolic homoeostasis, global deletion of $P \mathrm{~km} 2$ was recently shown to result in systemic inflammation in mice ${ }^{14}$. Our data indicate that inflammation associated with loss of PKM2 may, at least in part, be due to the de-repression of endogenous retroviral elements that trigger chronic activation of autoimmune signalling in both ECs and other cell types. More broadly, they indicate that metabolic dysfunction may be a trigger for autoimmune disease. The role of $\mathrm{NF}-\kappa \mathrm{B}$ in the regulation of immune signalling is well established $^{54}$. We have uncovered a homoeostatic function for RELB as a suppressor of ERV expression in ECs, which limits activation of antiviral innate immune signalling. The mechanisms through which RELB limits ERV expression remain to be identified, and could involve modifications to local chromatin composition or direct regulation of transcription (Supplementary Figure $7 \mathrm{~g}$ ). Examination of previously published microarray analysis of bone marrow dendritic cells from $\mathrm{Relb}^{-1-}$ mice ${ }^{45}$ shows that RELB limits ISG expression, likely by limiting ERV expression, in multiple cell types. Furthermore, Relb knockout mice display chronic inflammation characterized by immune cell infiltration into multiple tissues ${ }^{55}$, a phenotype we hypothesize may occur as a result of ERV de-repression. Collectively, these findings broaden our understanding of how endothelial metabolism is controlled and link metabolic changes with the control of growth, epigenetics and immune responses in ECs.

\section{Methods}

Cell culture. Human umbilical vein endothelial cells (HUVECs) were purchased from Lonza or Life Technologies, authenticated for EC marker expression and cultured until the fourth passage. HUVECs were cultured in endothelial basal medium-2 (EBM-2, Lonza) supplemented with foetal bovine serum, hydrocortisone, human basic fibroblast growth factor, vascular endothelial growth factor R3-insulin-like growth factor, ascorbic acid, human epidermal growth factor, GA1000 and heparin. Human embryonic kidney (HEK) cells were cultured in Dulbecco's modified Eagle's medium (DMEM) supplemented with $10 \%$ foetal calf serum (Thermo), $100 \mathrm{units} / \mathrm{ml}$ penicillin and $100 \mu \mathrm{g} / \mathrm{ml}$ streptomycin. Both cell lines were cultured in $5 \% \mathrm{CO}_{2}$ at $37^{\circ} \mathrm{C}$.

RNA interference. For pan-PKM or PKM2-specific silencing, siRNAs directed against $P K M$ or specifically targeting exon 10 of the $P K M$ gene have been described previously ${ }^{20}$. We validated the effect of three siRNA duplexes by assessing knockdown efficiency using RT-qPCR and western blot, and cell proliferation by quantifying EdU ${ }^{+}$cell numbers, glucose uptake, lactate and ATP levels. The following siRNA duplex was used for further studies: PKM2_si156:ccauaaucguccucaccaa. P53, RELA, RELB and REL were targeted with pools of siRNA duplexes (Mission esiRNA, Sigma). A negative control pool of four siRNAs (ON-TARGETplus Non-targeting pool, Dharmacon) was used to control for transfection. Transfections were performed using Lipofectamine RNAiMAX (Invitrogen) with the manufacturer's standard protocol. For all RNAi experiments, cells were reseeded $24 \mathrm{~h}$ after siRNA transfection to reach a confluence of $\sim 80 \%$ at $48 \mathrm{~h}$ after transfection.

RT-qPCR. RNA was isolated using the miRNeasy micro Kit (Qiagen) combined with on-column DNase digestion (DNase-Free DNase Set, Qiagen). An aliquot of $1 \mu \mathrm{g}$ of RNA was used for reverse transcription with the Maxima First Strand cDNA Synthesis Kit (Thermo). All PCR reactions were run on a CFX Connect Real-Time System (Biorad). The following primer pairs were used: PKM1_fwdCTATCCTCTGGAGGCTGTGC, $P K M 1$ rev-CCATGAGGTCTGTGGAGTGA; PKM2_fwd-CCACTTGCAATTATTTGAGGAA, PKM2_rev-GTGAGCAG ACCTGCCAGACT; TBP_fwd-GGAGAGTTCTGGGATTGTAC, TBP_revCTTATCCTCATGATTACCGCAG. For analysis of endogenous retrovirus expression, previously published primer pairs were used ${ }^{40}$. Expression levels were normalized to Tbp expression and fold changes calculated using the $\Delta \Delta \mathrm{Ct}$ method.

Western blot analysis and antibodies. HUVECs were lysed in modified RIPA buffer ( $150 \mathrm{~mm} \mathrm{NaCl}, 50 \mathrm{~mm}$ Tris- $\mathrm{HCl}$ pH 7.4, 1\% IGEPAL, $0.1 \%$ sodium deoxycholate, $1 \mathrm{~mm}$ EDTA) supplemented with protease inhibitors (cOmplete ULTRA Mini, Roche) and PMSF. Proteins were separated using SDS-PAGE on precast TGX gradient gels (Biorad) and then transferred to polyvinylidine fluoride membranes using the Transblot Turbo Transfer System (Bio-Rad). Membranes were blocked in 5\% BSA for $1 \mathrm{~h}$, probed with primary antibodies overnight at $4{ }^{\circ} \mathrm{C}$ and then with peroxidase-conjugated secondary antibodies. The following antibodies were used: PKM1 (Cell Signaling Technology, \#7067, 1:1000), PKM2 (Cell Signaling Technology, \#4053, 1:1000), P21 (Cell Signaling Technology, \#2947, 1:1000) P53 (Cell Signaling Technology, \#9282, 1:500), P53 (Santa Cruz, sc-126, 1:1000), RELB (Cell Signaling Technology, \#10544, 1:1000), MAT2A (Novus Biologicals, \#NB110-94158, 1:2000), $\beta$-actin (Cell Signaling Technology, \#8457, 1:1000), GAPDH (Cell Signaling Technology, \#2118, 1:2000) and anti-rabbit IgG-HRP (Santa Cruz Biotechnology, sc-2004, 1:10,000). For analysis, membranes were incubated with ECL (Clarity Western ECL Substrate, Biorad) and imaged using a ChemiDoc MP system (Biorad). Uncropped images of blots are presented in Supplementary Figure 8.

Seahorse metabolic analysis. Functional metabolic analyses in HUVECs were performed using a Seahorse XFe96 analyser (Seahorse Bioscience) according to the manufacturer's protocol. In brief, HUVECs (40,000 per well) were seeded on fibronectin-coated XFe96 microplates. After $2 \mathrm{~h}$, the cell medium was changed to a non-buffered assay medium and cells were maintained in a non- $\mathrm{CO}_{2}$ incubator for $1 \mathrm{~h}$. Using the Glycolysis Stress Test Kit, extracellular acidification rate (ECAR) was assessed under basal conditions, and sequentially following the addition of glucose $(10 \mathrm{mM})$, the mitochondrial ATP synthase inhibitor oligomycin $(3 \mu \mathrm{M})$ or 2 deoxy-D-glucose (2-DG; $100 \mathrm{mM}$ ), an inhibitor of glycolysis. Using the Mito Stress Test Kit, oxygen consumption rate (OCR) was measured sequentially under basal conditions, after injection of oligomycin $(3 \mu \mathrm{M})$, the mitochondrial uncoupler carbonyl cyanide-4-(trifluoromethoxy)phenyl-hydrazone (FCCP; $1 \mu \mathrm{M}$ ), and after 
injection of a mix of the respiratory chain inhibitors antimycin $\mathrm{A}(1.5 \mu \mathrm{M})$ and rotenone $(3 \mu \mathrm{M})$.

\begin{abstract}
Lactate assay. Lactate concentration was measured in HUVEC cell culture medium that was conditioned for $12 \mathrm{~h}$. The measurement was performed with a Lactate assay kit (Biovision) following the manufacturer's protocol.
\end{abstract}

Glucose uptake assay. Endothelial glucose uptake was assessed by analysing the uptake of 2-NBDG (2-(N-(7-Nitobenz-2oxa-1,3-diazol-4-yl)Amino)-2-Deoxyglucose) (Life Technologies), a fluorescent glucose analogue. HUVECs were incubated for $1 \mathrm{~h}$ in media containing $100 \mu \mathrm{M}$ 2-NBDG and fluorescence was measured using the BD FACS LSR II flow cytometer. Data were analysed with the BD FACSDiva software (Version 8.0.1).

Immunostaining of cell cultures. Twenty-four hours after transfection, HUVECs were re-seeded in 96-well imaging plates (Ibidi) to reach $\sim 80 \%$ confluence at $48 \mathrm{~h}$ after transfection. For EdU staining, cells were cultured in EGM2 media containing $10 \mu \mathrm{M}$ EdU (Thermo) for $3 \mathrm{~h}$ prior to fixation at $37^{\circ} \mathrm{C}$ and in $5 \% \mathrm{CO}_{2}$. Cells were fixed in 4\% PFA for $15 \mathrm{~min}$ at room temperature and then permeabilised with $0.5 \%$ Triton X-100 for $15 \mathrm{~min}$. To prevent antibody binding to non-specific epitopes, blocking was performed for $1 \mathrm{~h}$ in $5 \%$ goat serum, $1 \%$ BSA and $0.1 \%$ Triton X-100. Primary antibodies were diluted in blocking solution and incubated overnight at $4{ }^{\circ}$ C. The following primary antibodies were used: phospho-Histone H3 (Cell Signaling Technology, \#9713, 1:500) and J2A (Scicons, 1:200). Alexa Fluor-conjugated secondary antibodies were used for detection (Thermo, 1:250). The Click-iT EdU Alexa Fluor 647 imaging kit was used for detection of EdU incorporation (Thermo). Imaging was performed using an LSM800 Observer (Zeiss).

Cell cycle analyses. Control, $\mathrm{PKM} 22^{\mathrm{KD}}, \mathrm{PKM}{ }^{\mathrm{KD}}, \mathrm{P} 53^{\mathrm{KD}}$ and/or $\mathrm{PKM} 2{ }^{\mathrm{KD}} / \mathrm{P} 53^{\mathrm{KD}}$ HUVECs were harvested $48 \mathrm{~h}$ after transfection at $\sim 80 \%$ confluence. Adherent cells were fixed in ice-cold $70 \%$ ethanol and then stored at $-20^{\circ} \mathrm{C}$ overnight. For staining, cells were washed twice in PBS, resuspended in a solution of $50 \mathrm{mg} / \mathrm{ml}$ propidium iodide (Sigma-Aldrich) containing RNase (Sigma-Aldrich \#R6513) in $0.1 \%$ Triton X-100 and incubated for $20 \mathrm{~min}$ at $37^{\circ} \mathrm{C}$. Data were acquired using a BD FACS LSR II flow cytometer.

In vitro scratch wound assay. HUVECs were transfected with contol or PKM2 siRNAs and after $24 \mathrm{~h}$ seeded in 24 -well plates to reach $100 \%$ confluency at $48 \mathrm{~h}$ after transfection. At this stage, a scratch wound was applied to the EC monolayer using a $200 \mu$ l pipette tip, and individual wells were imaged $\left(T_{0}\right)$. HUVECs were incubated for a further $8 \mathrm{~h}$ in complete media, photographed again and the percentage wound closure in $\mathrm{ctrl}$ and $\mathrm{PKM} 2{ }^{\mathrm{KD}} \mathrm{ECs}$ calculated using ImageJ.

\section{Generation of zebrafish mutants with TALENs and CRISPR/Cas9. Animals} were maintained under standard conditions and all experiments were conducted in accordance with institutional (MPG) and national ethical and animal welfare guidelines. TALENs were used to generate $p k m a 2^{s 717}$ mutants and CRISPR/Cas9 was used to generate $p k m b^{s 718}$ mutants as previously described ${ }^{56}$. For the gen eration of pkma2 mutants, TALEN arms composed of the following RVDs targeting exon 10 of the pkma gene were used: NN NI HD HD HD NN NG NN NI HD HD HD NG NG HD NI NN NI NG NN HD and NG NG NN NI NI NN NN NI NI NN HD HD NG HD NN NI HD NN NN. RNA was synthesized with mMESSAGE mMachine (Ambion) and 50 pg per TALEN arm was injected into one-cell stage embryos to generate potential founders $\left(F_{0}\right)$. Analysis of mRNA expression in $p k m a 2^{+/+}$and $p k m a 2^{-/-}$embryos at $26 \mathrm{hpf}$ revealed no changes in pkma1 expression in pkma2 mutants (Supplementary Figure 2h). For CRISPR/ Cas9, sgRNAs were designed using the CRISPR Design online toolbox (http:// crispr.mit.edu) and the following guide sequence was used to target exon 5 of the $p k m b$ gene: TGTGGAACAGGGCGTGGACATGG. Guide RNAs were made by ligating annealed primers into the pT7-gRNA vector, and the pT3Ts-nCas9n plasmid (Addgene) was used to synthesize Cas9 mRNA. To generate founders, between $50 \mathrm{pg}$ of sgRNA and $250 \mathrm{pg}$ of Cas 9 mRNA were coinjected into one-cell stage embryos. $F_{0}$ founders for each gene were identified by outcrossing TALEN or CRISPR/Cas9-injected fish with $\mathrm{AB}$ and screening the offspring at $24 \mathrm{hpf}$ using HRMA. PCR products from $F_{0}$ founders that showed promising HRMA melt curves were cloned and sequenced, and those with frameshift mutations were grown. Primer sequences for HRMA genotyping were as follows: pkma2_HRMA_fwd: CTGTTCTGACCCGTGACCCTTC; pkma2_HRMA_rev: GGTGAG TATGATGATGCCGC; $p k m b \_H R M A$ fwd: CTGAGAAGGACATCAAGGACC; pkmb_HRMA_rev: AGCAGCTTTGCGGATAAAGGAGG. Mutants were generated in the $T g(k d r l: E G F P)^{s 843}$ background $^{57}$.

Mouse experiments. Animals were maintained under standard conditions and all experiments were conducted in accordance with institutional (MPG) and national ethical and animal welfare guidelines. Conditional PKM2 mutant mice $\left(P \mathrm{~km}^{\text {tm1.1Mgvh}}\right)$ were backcrossed to the C57/Bl6 background. For conditional ECspecific deletion, PKM2-floxed mice were bred with $T g(P d g f b \text {-icre/ERT2 })^{1 \text { Frut }}$ mice, which express the tamoxifen-inducible CreERT2 in ECs. For analysis of angiogenesis in the retina, postnatal mice were injected intraperitoneally with $25 \mu \mathrm{l}$ of 4 Hydoxytamoxifen $(2 \mathrm{mg} / \mathrm{ml}, \mathrm{H} 6278$, Sigma) on postnatal days (P)1-P3 and retinas were harvested on P7. Control animals were littermates without CreERT2 expression. Due to the nature of the experimental setup, animals were randomly assigned to treatment groups. Only litters that reached normal body weight at P7 were used. Mice that were more than 1.5 interquartile ranges (IQR) above or below the upper or lower IQR for their respective litter were not analysed.

Immunostaining and analysis of postnatal retinas. For quantitative analysis of angiogenesis, eyes were harvested from P7 mice and fixed in 4\% PFA on ice for $2 \mathrm{~h}$ After fixation, eyes were washed in PBS and then dissected to remove the retinal tissue. Following dissection, retinas were incubated in blocking buffer (3\% normal goat serum, $1 \%$ BSA, $0.5 \%$ Triton X-100 and $0.5 \%$ Tween-20) for $1 \mathrm{~h}$ at RT and then incubated with primary antibodies diluted in incubation buffer $(1.5 \%$ normal goat serum, $0.5 \%$ BSA, $0.25 \%$ Triton X-100 and $0.25 \%$ Tween-20) overnight at $4{ }^{\circ} \mathrm{C}$. Primary antibodies against the following proteins were used: PECAM (1:200, \#AF3628, R\&D Systems), ERG (1:200, \#ab92513, Abcam), Collagen IV (1:400, $\# 2150-1470$, AbD Serotec). After primary antibody incubation, retinas were washed four times in $0.1 \%$ PBS Triton X-100 and incubated with Alexa Fluor-conjugated secondary antibodies (Thermo, 1:250) for $2 \mathrm{~h}$ at RT. Following secondary antibody staining, retinas were washed four times in $0.1 \%$ PBS Triton X-100 and the ClickiT EdU Alexa Fluor 647 imaging kit was used for detection of EdU incorporation (Thermo). Retinas were mounted on glass slides using Vectashield (Vector Labs) and imaging was performed using a Zeiss LSM700 or Leica SP8 microscope. All images were acquired without prior knowledge of the genotype of the individual samples.

Quantitative analysis of the retinal vasculature. Outgrowth of the retinal vasculature was measured using ImageJ software as the distance from the centre of the optic nerve to the periphery of the vessel bed in each leaflet of dissected retinas. The mean distance of individual leaflets was taken as the value for each sample. For quantification of EC and EdU ${ }^{+} \mathrm{EC}$ number, vessel area, branch point density and empty Collagen IV sleeves, four fields per sample were acquired using a $\times 25$ objective. The total number of ECs and $\mathrm{EdU}^{+} \mathrm{EC}$ number was determined using Imaris Software. The mean of four fields per sample at the angiogenic front or central plexus was taken as the value for each sample. EC area per field and branch point density was determined using the AngioTool software. Empty Collagen IV sleeves were counted manually using ImageJ. These analyses were not blinded.

LC-HRMS metabolomics and flux analysis. For measurement of steady-state metabolite levels, control, $\mathrm{PKM} 2^{\mathrm{KD}}, \mathrm{PKM}^{\mathrm{KD}}, \mathrm{P} 53^{\mathrm{KD}}$ and/or $\mathrm{PKM} 2^{\mathrm{KD}} / \mathrm{P} 53^{\mathrm{KD}}$ HUVECs were harvested $48 \mathrm{~h}$ after transfection in $80 \%$ methanol pre-cooled to $-80^{\circ} \mathrm{C}$. Plates were transferred to a $-80^{\circ} \mathrm{C}$ freezer for $15 \mathrm{~min}$ to inactivate enzyme functions, then scraped into extraction solvent and centrifuged at $20,000 \times g$ for $10 \mathrm{~min}$ at $4{ }^{\circ} \mathrm{C}$. The supernatant was then collected and dried for $\sim 2 \mathrm{~h}$ in a speed$\mathrm{vac}$ at room temperature. Dried pellets were stored at $-80^{\circ} \mathrm{C}$. For flux analyses, 50:50 labelling was performed using [U-13C 6 ]glucose (Cambridge Isotope Laboratories, Inc) as a tracer, and metabolite extraction and storage was performed as above. Metabolomics was performed using liquid chromatography-high resolution mass spectrometry (LC-HRMS). An ultimate 3000 UHPLC (Dionex) is coupled to the Q Exactive Plus-Mass spectrometer (QE-MS, Thermo Scientific, San Jose, CA) for metabolite separation and detection. Detailed instrument method information was described previously ${ }^{58}$. Samples were reconstituted into $30 \mu \mathrm{l}$ sample solvent (water:methanol:acetonitrile, $2: 1: 1, \mathrm{v} / \mathrm{v}$ ), and $4 \mu \mathrm{l}$ was injected to the LC-QE-MS. The peak area of each metabolite was used to present relative abundance and to calculate the isotopologue distribution. All data are provided in Supplementary Data File 1.

Proteomics analysis. Control and PKM2 ${ }^{\mathrm{KD}}$ HUVECs were harvested in $4 \%$ SDS $0.1 \mathrm{M}$ Tris- $\mathrm{HCl}$ (pH 7.6) $48 \mathrm{~h}$ after transfection. Samples were sonicated, clarified by centrifugation and protein concentration was estimated using the DCA protein assay kit. Proteins were separated using SDS-PAGE on precast TGX gradient gels (Bio-Rad) and stained using the Colloidal Blue staining kit (Invitrogen). Each lane of the gel was cut into slices, and proteins were digested as previously described ${ }^{56}$ Briefly, gel pieces were washed with $50 \mathrm{mM}$ ammoniumbicarbonate and ethanol, reduced with $10 \mathrm{mM}$ DTT for $45 \mathrm{~min}$ at $56^{\circ} \mathrm{C}$, carbamidomethylated with $55 \mathrm{mM}$ iodoacetamide for $30 \mathrm{~min}$ at RT in the dark, and then digested with trypsin overnight. The next day, peptides were extracted from the gel pieces using increasing concentrations of acetonitrile. Samples were cleaned up with Stage Tips (Stop and go extraction) and then measured with LC-MS/MS on a QExactive HF Separation of peptides according to their hydrophobicities was achieved on a $15 \mathrm{~cm}$ in-house packed column (internal diameter $75 \mu \mathrm{m}, \mathrm{C} 18$ Beads (Dr. Maisch) diameter $1.8 \mu \mathrm{m}$ ) using a binary buffer system: (A) $0.1 \%$ formic acid in $\mathrm{H}_{2} \mathrm{O}$ and (B) $0.1 \%$ formic acid in $80 \%$ acetonitrile. The following gradient of 75 min was used: $5 \mathrm{~min} 10 \%$ of B, $50 \mathrm{~min} 38 \%$ of B, 5 min $60 \%$ of B, $10 \min 95 \%$ of B, 5 min $5 \%$ of B. Mass spectra were acquired at a resolution of $60,000(200 \mathrm{~m} / \mathrm{z})$ using an AGC target of $3 \mathrm{e} 6$ and a maximal injection time of $20 \mathrm{~ms}$. A top 15 method was applied for subsequent acquisition of high-energy collision-induced dissociation (HCD) 
fragmentation MS/MS spectra of the 15 most intense peaks. Fragmentation (MS/ MS) was performed with a resolution of 15,000 at $2.2 \mathrm{~m} / z$ and and AGC target of 1e5 within a maximal injection time of $25 \mathrm{~ms}$. Raw files were processed using MaxQuant 1.5.5.1 and the Andromeda search engine. For peptide assignment, MS/ MS HCD fragmentation spectra were correlated to the Uniprot Homo sapiens database (2016). Further downstream analyses were performed as previously described. All data are provided in Supplementary Data File 2.

RNA-seq analysis and data processing. RNA was isolated from $48 \mathrm{~h}$ after transfection from control, $\mathrm{P} 53^{\mathrm{KD}}, \mathrm{PKM} 2^{\mathrm{KD}}$ and $\mathrm{PKM} 2^{\mathrm{KD}} / \mathrm{P} 53^{\mathrm{KD}}$ HUVECs using the miRNeasy micro Kit (Qiagen) combined with on-column DNase digestion (DNase-Free DNase Set, Qiagen). RNA and library preparation integrity were verified with a BioAnalyzer 2100 (Agilent) or LabChip Gx Touch 24 (Perkin Elmer). $2 \mu \mathrm{g}$ of total RNA was used as input for Truseq Stranded mRNA Library preparation following the low sample protocol (Illumina). Sequencing was performed on the NextSeq500 instrument (Illumina) using v2 chemistry, resulting in a minimum of $37 \mathrm{M}$ reads per library with $1 \times 75 \mathrm{bp}$ single end setup. The resulting raw reads were assessed for quality, adaptor content and duplication rates with FastQC ${ }^{59}$. Reaper version 13-100 was employed to trim reads after a quality drop below a mean of Q20 in a window of ten nucleotides ${ }^{60}$. Only reads between 30 and 150 nucleotides were cleared for further analyses. Trimmed and filtered reads were aligned to the GRCh37.p5 human genome assembly using STAR 2.4.0a with the parameter "--outFilterMismatchNoverLmax 0.1 " to increase the maximum ratio of mismatches to mapped length to $10 \%{ }^{61}$. The number of reads aligning to genes was counted with the featureCounts 1.4.5-p1 tool from the Subread package ${ }^{62}$. Only reads mapping at least partially inside exons were admitted and aggregated per gene. Reads overlapping multiple genes or aligning to multiple regions were excluded. Differentially expressed genes were identified using DESeq2 version $1.62^{63}$.

5-methylcytosine quantification. The levels of 5-methylcytosine were measured using the MethylFlash Methylated DNA quantification kit (Epigenetik) according to the instructions of the manufacturer.

Chromatin immunoprecipitation (ChIP). ChIP was performed using the truChIP Chromatin Shearing Reagent kit (Covaris) according to the manufacturer's instructions. Chromatin was sheared (Bioruptor, Diagenode) to generate fragments between 200 and 400 bp. $5 \mu \mathrm{g}$ of DNA was used for each immunoprecipitation and $10 \%$ was stored as an input. Immunoprecipitation was performed following a published protocol ${ }^{64}$ using the following antibodies: Mouse IgG $(2 \mu \mathrm{g} / \mathrm{IP}, 5441 \mathrm{~S}$, Cell Signaling) and Histone H3 (tri methyl K9) ( $2 \mu \mathrm{g} / \mathrm{IP}$, ab8898, Abcam). Following immunoprecipitation and reverse cross-linking, samples and inputs were purified using the NucleoSpin Gel and PCR Clean-up kit (Macherey-Nagel) following the manufacturer's instructions for samples containing SDS.

Bisulfite conversion and restriction analyses. Genomic DNA was isolated from $80 \%$ confluent control, $\mathrm{PKM} 2^{\mathrm{KD}}, \mathrm{P} 53^{\mathrm{KD}}, \mathrm{PKM} 2^{\mathrm{KD}} / \mathrm{P} 53^{\mathrm{KD}}, \mathrm{RELB}{ }^{\mathrm{KD}}$ and PKM $2^{\mathrm{KD}}$ / RELB $^{\mathrm{KD}}$ HUVECs $48 \mathrm{~h}$ after transfection, using the DNeasy Blood \& Tissue kit (Qiagen, 69504). Bisulfite conversion was performed using the EpiJET Bisulfite Conversion Kit (Thermo Scientific, \#K1461) according to the manufacturer's instructions. PCR amplification of bisulfite-converted DNA was performed using Phusion U Hot Start DNA Polymerase (Thermo Scientific, F555S) with the following primers: $M L T 1 B \_F w d-A G T T A T A G A T A G G T A T A G A G T G T T G G T T T A$ TAG; $M L T 1 B \_R e v-C C T A T T C T A A C C A T T T T C T A T C A A T A A A A T C ;$ MER4D_Fwd-TTTGAGGAAGTGTGTTTGAGGTTGTTAGGATG; MER4D_Rev-CCTCTAACACTACCTTAACTCAAACATTTC. Following PCR amplification, samples were purified and then digested with HpyCH4III (MER4D) or HincII $(M L T 1 B)$ for $8 \mathrm{~h}$, and visualized on a $3 \%$ agarose gel.

Blinding. The individual genotypes of mice and zebrafish were unknown before data analysis was performed.

Statistical analyses. No statistical tests were used to predetermine sample size Statistical analyses were performed by unpaired, two-tailed Student's $t$ test, or nonparametric one-way ANOVA followed by Tukeys HSD test using GraphPad Prism software. Data are represented as mean \pm s.d. for individual data points and mean \pm s.e.m. where values presented are calculated as a mean of means. $P$ values $<0.05$ were considered significant. All metabolomic (Figs. 1c-l, 4b, 5f, 6b, and Supplementary Figures 1e-j, 5f-g, 6a), transcriptomic (Figs. 4c, 6d and Supplementary Figures 4a, e, 5a, 6b), proteomic (Fig. 5e and Supplementary Figures 5b, 6c, 7b), RT-qPCR (Figs. 1a, 6g, i and Supplementary Figures 1c, 7b), ChIP RT-qPCR (Supplementary Figure $7 \mathrm{a}, \mathrm{f}$ ) and phenotypic (Figs. 2a-c, 5a, c and Supplementary Figures $1 \mathrm{a}-\mathrm{c}, 5 \mathrm{c}, \mathrm{d}$ ) analyses were performed by transfecting primary HUVECs pooled from multiple donors ( $n=$ number of independent transfections). For in vivo analyses in zebrafish (Fig. 2d, e) and mouse (Fig. 3b, d and Supplementary Figure $3 \mathrm{e}), n=$ number of independent animals observed. Sample sizes were selected on the basis of published protocols ${ }^{65}$.

\section{Data availability}

RNA-seq data are deposited in the Gene Expression Omnibus under accession number GSE84877. Metabolomics (Supplementary Data File 1) and proteomics (Supplementary Data File 2) data are provided as supplementary data files.

Received: 27 September 2017 Accepted: 1 September 2018 Published online: 09 October 2018

\section{References}

1. Potente, M., Gerhardt, H. \& Carmeliet, P. Basic and therapeutic aspects of angiogenesis. Cell 146, 873-887 (2011).

2. De Bock, K. et al. Role of PFKFB3-driven glycolysis in vessel sprouting. Cell 154, 651-663 (2013).

3. Kim, B., Li, J., Jang, C. \& Arany, Z. Glutamine fuels proliferation but not migration of endothelial cells. EMBO J. 36, 2321-2333 (2017).

4. Cruys, B. et al. Glycolytic regulation of cell rearrangement in angiogenesis. Nat. Commun. 7, 12240 (2016).

5. De Bock, K., Georgiadou, M. \& Carmeliet, P. Role of endothelial cell metabolism in vessel sprouting. Cell Metab. 18, 634-647 (2013).

6. Schoors, S. et al. Partial and transient reduction of glycolysis by PFKFB3 blockade reduces pathological angiogenesis. Cell Metab. 19, 37-48 (2014).

7. Quintero, M., Colombo, S. L., Godfrey, A. \& Moncada, S. Mitochondria as signaling organelles in the vascular endothelium. Proc. Natl Acad. Sci. USA 103, 5379-5384 (2006).

8. Schoors, S. et al. Fatty acid carbon is essential for dNTP synthesis in endothelial cells. Nature 520, 192-197 (2015)

9. Huang, $\mathrm{H}$. et al. Role of glutamine and interlinked asparagine metabolism in vessel formation. EMBO J. 36, 2321-2333 (2017).

10. Anastasiou, D. et al. Pyruvate kinase M2 activators promote tetramer formation and suppress tumorigenesis. Nat. Chem. Biol. 8, 839-847 (2012).

11. Christofk, H. R. et al. The M2 splice isoform of pyruvate kinase is important for cancer metabolism and tumour growth. Nature 452, 230-233 (2008).

12. Qi, W. et al. Pyruvate kinase M2 activation may protect against the progression of diabetic glomerular pathology and mitochondrial dysfunction. Nat. Med. 23, 753-762 (2017).

13. Mazurek, S., Boschek, C. B., Hugo, F. \& Eigenbrodt, E. Pyruvate kinase type $\mathrm{M} 2$ and its role in tumor growth and spreading. Semin. Cancer Biol. 15, 300-308 (2005)

14. Dayton, T. L. et al. Germline loss of PKM2 promotes metabolic distress and hepatocellular carcinoma. Genes Dev. 30, 1020-1033 (2016).

15. Wang, Z. et al. Exon-centric regulation of pyruvate kinase $M$ alternative splicing via mutually exclusive exons. J. Mol. Cell Biol. 4, 79-87 (2012).

16. Keller, K. E., Tan, I. S. \& Lee, Y. S. SAICAR stimulates pyruvate kinase isoform M2 and promotes cancer cell survival in glucose-limited conditions. Science 338, 1069-1072 (2012).

17. Ikeda, Y. \& Noguchi, T. Allosteric regulation of pyruvate kinase M2 isozyme involves a cysteine residue in the intersubunit contact. J. Biol. Chem. $\mathbf{2 7 3}$ 12227-12233 (1998).

18. Chaneton, B. et al. Serine is a natural ligand and allosteric activator of pyruvate kinase M2. Nature 491, 458-462 (2012).

19. Christofk, H. R., Vander Heiden, M. G., Wu, N., Asara, J. M. \& Cantley, L. C. Pyruvate kinase M2 is a phosphotyrosine-binding protein. Nature $\mathbf{4 5 2}$ 181-186 (2008).

20. Goldberg, M. S. \& Sharp, P. A. Pyruvate kinase M2-specific siRNA induces apoptosis and tumor regression. J. Exp. Med. 209, 217-224 (2012).

21. Santoro, M. M. Fashioning blood vessels by ROS signalling and metabolism. Semin. Cell Dev. Biol. 80, 35-42 (2017).

22. del Toro, R. et al. Identification and functional analysis of endothelial tip cellenriched genes. Blood 116, 4025-4033 (2010)

23. Bao, X. R. et al. Mitochondrial dysfunction remodels one-carbon metabolism in human cells. eLife 5, e10575 (2016).

24. Locasale, J. W. Serine, glycine and one-carbon units: cancer metabolism in full circle. Nat. Rev. Cancer 13, 572-583 (2013).

25. Lunt, S. Y. et al. Pyruvate kinase isoform expression alters nucleotide synthesis to impact cell proliferation. Mol. Cell 57, 95-107 (2015).

26. Berkers, C. R., Maddocks, O. D., Cheung, E. C., Mor, I. \& Vousden, K. H. Metabolic regulation by p53 family members. Cell Metab. 18, 617-633 (2013).

27. Kawauchi, K., Araki, K., Tobiume, K. \& Tanaka, N. p53 regulates glucose metabolism through an IKK-NF-kappaB pathway and inhibits cell transformation. Nat. Cell Biol. 10, 611-618 (2008).

28. Mauro, C. et al. NF-kappaB controls energy homeostasis and metabolic adaptation by upregulating mitochondrial respiration. Nat. Cell Biol. 13, 1272-1279 (2011) 
29. Johnson, R. F. \& Perkins, N. D. Nuclear factor-kappaB, p53, and mitochondria: regulation of cellular metabolism and the Warburg effect. Trends Biochem. Sci. 37, 317-324 (2012).

30. Levine, A. J. \& Oren, M. The first 30 years of p53: growing ever more complex. Nat. Rev. Cancer 9, 749-758 (2009).

31. Maddocks, O. D. et al. Serine starvation induces stress and p53-dependent metabolic remodelling in cancer cells. Nature 493, 542-546 (2013).

32. Gut, P. \& Verdin, E. The nexus of chromatin regulation and intermediary metabolism. Nature 502, 489-498 (2013).

33. Mehrmohamadi, M., Mentch, L. K., Clark, A. G. \& Locasale, J. W. Integrative modelling of tumour DNA methylation quantifies the contribution of metabolism. Nat. Commun. 7, 13666 (2016).

34. Caudill, M. A. et al. Intracellular S-adenosylhomocysteine concentrations predict global DNA hypomethylation in tissues of methyl-deficient cystathionine beta-synthase heterozygous mice. J. Nutr. 131, 2811-2818 (2001).

35. Wu, S. C. \& Zhang, Y. Active DNA demethylation: many roads lead to Rome. Nat. Rev. Mol. Cell Biol. 11, 607-620 (2010).

36. Lander, E. S. et al. Initial sequencing and analysis of the human genome. Nature 409, 860-921 (2001).

37. Cordaux, R. \& Batzer, M. A. The impact of retrotransposons on human genome evolution. Nat. Rev. Genet. 10, 691-703 (2009).

38. Radoshevich, L. \& Dussurget, O. Cytosolic Innate Immune Sensing and Signaling upon Infection. Front. Microbiol. 7, 313 (2016).

39. Chiappinelli, K. B. et al. Inhibiting DNA methylation causes an interferon response in cancer via dsRNA including endogenous retroviruses. Cell 162, 974-986 (2015).

40. Roulois, D. et al. DNA-demethylating agents target colorectal cancer cells by inducing viral mimicry by endogenous transcripts. Cell 162, 961-973 (2015).

41. Barbalat, R., Ewald, S. E., Mouchess, M. L. \& Barton, G. M. Nucleic acid recognition by the innate immune system. Annu. Rev. Immunol. 29, 185-214 (2011).

42. Rowe, H. M. \& Trono, D. Dynamic control of endogenous retroviruses during development. Virology 411, 273-287 (2011).

43. Leonova, K. I. et al. p53 cooperates with DNA methylation and a suicidal interferon response to maintain epigenetic silencing of repeats and noncoding RNAs. Proc. Natl Acad. Sci. USA 110, E89-E98 (2013).

44. Iwasaki, A. \& Pillai, P. S. Innate immunity to influenza virus infection. Nat. Rev. Immunol. 14, 315-328 (2014).

45. Shih, V. F. et al. Control of RelB during dendritic cell activation integrates canonical and noncanonical NF-kappaB pathways. Nat. Immunol. 13 1162-1170 (2012).

46. Puto, L. A. \& Reed, J. C. Daxx represses RelB target promoters via DNA methyltransferase recruitment and DNA hypermethylation. Genes Dev. 22, 998-1010 (2008).

47. Yang, W. et al. Nuclear PKM2 regulates beta-catenin transactivation upon EGFR activation. Nature 480, 118-122 (2011).

48. Israelsen, W. J. et al. PKM2 isoform-specific deletion reveals a differential requirement for pyruvate kinase in tumor cells. Cell 155, 397-409 (2013).

49. Reid, M. A., Dai, Z. \& Locasale, J. W. The impact of cellular metabolism on chromatin dynamics and epigenetics. Nat. Cell Biol. 19, 1298-1306 (2017).

50. Carey, B. W., Finley, L. W., Cross, J. R., Allis, C. D. \& Thompson, C. B. Intracellular alpha-ketoglutarate maintains the pluripotency of embryonic stem cells. Nature 518, 413-416 (2015).

51. Mentch, S. J. et al. Histone methylation dynamics and gene regulation occur through the sensing of one-carbon metabolism. Cell Metab. 22, 861-873 (2015).

52. $\mathrm{Li}, \mathrm{S}$. et al. Serine and SAM responsive complex SESAME regulates histone modification crosstalk by sensing cellular metabolism. Mol. Cell 60, 408-421 (2015).

53. Chiappinelli, K. B. et al. Inhibiting DNA methylation causes an interferon response in cancer via dsRNA including endogenous retroviruses. Cell 164, 1073 (2016).

54. Hayden, M. S. \& Ghosh, S. NF-kappaB, the first quarter-century: remarkable progress and outstanding questions. Genes Dev. 26, 203-234 (2012).

55. Weih, F. et al. Multiorgan inflammation and hematopoietic abnormalities in mice with a targeted disruption of RelB, a member of the NF-kappa B/Rel family. Cell 80, 331-340 (1995).

56. Reischauer, $\mathrm{S}$. et al. Cloche is a bHLH-PAS transcription factor that drives haemato-vascular specification. Nature 535, 294-298 (2016).

57. Jin, S. W., Beis, D., Mitchell, T., Chen, J. N. \& Stainier, D. Y. Cellular and molecular analyses of vascular tube and lumen formation in zebrafish. Development 132, 5199-5209 (2005).
58. Liu, X., Ser, Z. \& Locasale, J. W. Development and quantitative evaluation of a high-resolution metabolomics technology. Anal. Chem. 86, 2175-2184 (2014).

59. Andrews, S. FastQC: a quality control tool for high throughput sequence data (Babraham Bioinformatics, 2010).

60. Davis, M. P., van Dongen, S., Abreu-Goodger, C., Bartonicek, N. \& Enright, A. J. Kraken: a set of tools for quality control and analysis of high-throughput sequence data. Methods 63, 41-49 (2013).

61. Dobin, A. et al. STAR: ultrafast universal RNA-seq aligner. Bioinformatics 29, 15-21 (2013).

62. Liao, Y., Smyth, G. K. \& Shi, W. featureCounts: an efficient general purpose program for assigning sequence reads to genomic features. Bioinformatics $\mathbf{3 0}$, 923-930 (2014).

63. Love, M. I., Huber, W. \& Anders, S. Moderated estimation of fold change and dispersion for RNA-seq data with DESeq2. Genome Biol. 15, 550 (2014).

64. Blecher-Gonen, R. et al. High-throughput chromatin immunoprecipitation for genome-wide mapping of in vivo protein-DNA interactions and epigenomic states. Nat. Protoc. 8, 539-554 (2013).

65. Pitulescu, M. E., Schmidt, I., Benedito, R. \& Adams, R. H. Inducible gene targeting in the neonatal vasculature and analysis of retinal angiogenesis in mice. Nat. Protoc. 5, 1518-1534 (2010).

\section{Acknowledgements}

We thank Matt Vander Heiden for $P k m 2^{f l / f l}$ mice and Marcus Fruttiger for $P d g f b$ CreERT2 mice, and Philipp Gut for discussions and critical reading of the manuscript. This work was supported by the Excellence Cluster Cardiopulmonary System (ECCPS EXC147, DFG) (O.A.S.), the European Research Council Starting Grant ANGIOMET (311546) (M.P.), the Deutsche Forschungsgemeinschaft (SFB 834) (M.P. and D.Y.R.S.), the German Center for Cardiovascular Research DZHK (BMBF) (T.B., M.P. and D.Y.R S.), the LOEWE grant Ub-Net (M.P.), the European Molecular Biology Organization Young Investigator Programme (M.P.), the Packard Foundation (D.Y.R.S.) and the Max Planck Society (T.B., M.P. and D.Y.R.S.).

\section{Author contributions}

Conceived and directed the study (O.A.S. and D.Y.R.S.), performed experiments (O.A.S., M.E.-B., K.W., X.L., A.M.R., E.G., S.G., S.J. and I.-C.L.), bioinformatics (J.K.H.L., S.G. and C.K.), supervised experiments (O.A.S., T.B., M.S., J.L., M.P. and D.Y.R.S.), wrote the paper (O.A.S.). All authors contributed to data analysis and commented on the paper.

\section{Additional information}

Supplementary Information accompanies this paper at https://doi.org/10.1038/s41467018-06406-8.

Competing interests: The authors declare no competing interests.

Reprints and permission information is available online at http://npg.nature.com/ reprintsandpermissions/

Publisher's note: Springer Nature remains neutral with regard to jurisdictional claims in published maps and institutional affiliations.

cc) (i) Open Access This article is licensed under a Creative Commons BY Attribution 4.0 International License, which permits use, sharing, adaptation, distribution and reproduction in any medium or format, as long as you give appropriate credit to the original author(s) and the source, provide a link to the Creative Commons license, and indicate if changes were made. The images or other third party material in this article are included in the article's Creative Commons license, unless indicated otherwise in a credit line to the material. If material is not included in the article's Creative Commons license and your intended use is not permitted by statutory regulation or exceeds the permitted use, you will need to obtain permission directly from the copyright holder. To view a copy of this license, visit http://creativecommons.org/ licenses/by/4.0/.

(C) The Author(s) 2018 